Abstracts*)

\title{
2nd European Conference on Diagnostic Error in Medicine August 30-31, 2018, Bern, Switzerland
}

\author{
Scientific Committee \\ David Schwappach, MD, MPH, Patient Safety Switzerland, Zurich, Switzerland \\ Jason Maude, Isabel Healthcare, Haslemere, United Kingdom \\ Juliane Kämmer, PhD, Max Planck Institute for Human Development and Charité University Medicine, Berlin, Germany \\ Laura Zwaan, PhD, Co-Chair, Institute for Medical Education Research, Erasmus Medical Center, Rotterdam, Netherland \\ Maarten ten Berg, Department of Clinical Chemistry and Haematology, University Medical Center Utrecht, Netherland \\ Wolf Hautz, MD, MME, Chair, Department of Emergency Medicine, Inselspital University Hospital Bern, Bern, Switzerland
}

\section{Organization}

Department of Emergency Medicine, Inselspital University Hospital Bern, Switzerland

Ms. Sascha Fischer

and

BBS Congress GmbH

\footnotetext{
*) These abstracts have been reproduced directly from the material supplied by the authors, without editorial alteration by the staff of this Journal. Insufficiencies of preparation, grammar, spelling, style, syntax, and usage are the authors.
} 


\section{Scientific Abstracts}

\section{Plenary Oral}

Thursday August 30, 2018, 14.00-15.30h

1.

\section{Clinician Communication and Data Sharing during the Process of Diagnosis in the Inpatient Setting}

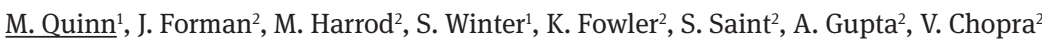

${ }^{1}$ University of Michigan, Ann Arbor, MI

${ }^{2}$ VA Ann Arbor Healthcare System, Ann Arbor, MI

Background: The process of formulating a working diagnosis in the inpatient setting requires that diagnosticians working in complex and hectic hospital environments gather, filter, integrate, and interpret substantial amounts of information within a short time. Several years ago, the information needed for diagnosis was gathered primarily at the bedside. More recently and with the advance of new technologies, this information is collected through a series of communication exchanges (e.g., pagers, emails) and interactions with the electronic health record (EHR). In this study, we examined challenges and opportunities for improvement in clinician-to-clinician communication and data sharing during the diagnostic process.

Methods: We performed a qualitative, multi-method, focused ethnographic study. Data were gathered between January and May 2016 at two affiliated teaching hospitals. Eight inpatient medicine teams (which included attending physicians, senior residents, interns, and medical students) were observed during morning rounds and in the afternoon on call and non-call days. Focus groups and interviews were then conducted with team members to better understand challenges and opportunities for improvement. Unstructured field notes were taken during observations. All focus groups and interviews were recorded and transcribed. Data were analyzed using qualitative content analysis.

Results: Observation data showed that physicians faced a data-gathering and communication environment that made integration and interpretation of information for diagnosis challenging. Notably, data flow and communication for each patient was fragmented over time and diagnostic information was pieced together from multiple sources. Pagers were inefficient and did not support dialogue needed for diagnosis. Suggestions for improvement during interviews and focus groups included: 1) replacing pagers with two-way communication technologies; 2) improving EHR design to support diagnosis by increasing data integration while reducing data overload and information fragmentation; 3) identifying more efficient ways to access the EHR during morning rounds and while in patient rooms; 4) increasing face-to-face communication between clinicians. Conclusion: Teaching hospitals are complex environments. The way patient information is shared and communicated among clinicians has changed with the adoption of electronic health records. Physicians are confronted with data overload, frequent interruptions, fragmented information, and little time to think about diagnosis. Although improvement opportunities suggested by front line physicians for patient diagnosis were identified, how best to implement these ideas remains to be determined. 
2.

Diagnostic Error and Information Distortion

\author{
M. Nurek ${ }^{1}$, M. A. Vadillo ${ }^{2}$, O. Kostopoulou ${ }^{1}$ \\ ${ }^{1}$ Imperial College London, London, United Kingdom \\ ${ }^{2}$ Universidad Autónoma de Madrid, Madrid, Spain
}

Background: Our inherent drive to formulate coherent judgements can lead to biased information processing: we may distort new evidence to favour a pre-existing belief, before reaching a final judgement and/or decision. This phenomenon - known as "information distortion" (or simply "distortion") - has also been identified in diagnosis: physicians may interpret patient information in a way that favours their leading diagnostic hypothesis. We measured the contribution of this cognitive bias to diagnostic error.

Methods: We constructed two patient cases, each with two competing diagnoses. One diagnosis was common and non-serious, the other less common and serious. Each case consisted of a brief patient description (demographics and health complaint) and several cues (symptoms, signs, and investigation results). Based on the available cues, the serious diagnoses could not be ruled out and warranted specialist referral. We presented 148 family physicians with one of the two patient cases, at random. After reading the patient description, participants chose one of the two competing diagnoses. They then elicited further information: cues were arranged as labelled buttons on an information board that participants could click to reveal the answer. Each time that a cue was revealed, participants evaluated it in relation to each competing diagnosis ( $0=$ "no support" to $10=$ "strong support") and updated their diagnostic choice. When they felt ready, they made their final choice of diagnosis. To measure distortion, we compared these "biased" cue evaluations to the "unbiased" evaluations provided by a separate control group of physicians, who rated the same cues but each in isolation (i.e., not in the context of a coherent patient case). We measured the contribution of distortion to the final diagnosis, using mediation analysis.

Results: Initial choice of diagnosis (non-serious vs. serious) predicted final choice $(\mathrm{OR}=4.78, P<0.001)$. Distortion mediated this relationship fully: initial selection of the non-serious diagnosis was associated with distortion in its favour $(B=2.37, P<0.001)$, which increased the odds of selecting the non-serious diagnosis in the end $(\mathrm{OR}=3.07, P<0.001)$. Final diagnosis predicted management: most physicians who selected the non-serious diagnosis did not refer the patient (70\%), whilst only $3 \%$ of those who selected the serious diagnosis failed to refer (chi-square $=71.62, \mathrm{df}=1, P<0.001$ ).

Conclusion: Initial diagnostic hypotheses are important for the final outcome of the diagnostic process, as they can bias the interpretation of subsequent information. This is the first study to demonstrate that distorting new clinical information in favour of an initial diagnosis can result in misdiagnosis. 
3.

\title{
How Available Is Availability? a Study to Identify Factors That Influence Reliance on Available Information
}

\author{
$\underline{\text { S. Monteiro }}^{1}$, J. Sherbino ${ }^{1}$, G. Norman ${ }^{1}$, J. Ilgen ${ }^{2}$, E. Hayden ${ }^{3}$ \\ ${ }^{1}$ McMaster University, Hamilton, ON, Canada \\ ${ }^{2}$ University of Washington, Seattle, WA \\ ${ }^{3}$ Harvard Medical School, Boston, MA
}

Background: Cognitive bias is often cited as an explanation for diagnostic errors. Availability bias, defined as "The current case reminds you of a recent similar example or diagnostic category" is most well-known. Despite the ubiquity of availability bias in medical and popular literature, there is surprisingly little evidence to substantiate these claims. The present study sought to measure the influence of availability bias. Methods: We devised a sequential 2 phase experiment. Phase 2, the test phase, consisted of 8 written cases, each with two approximately equally likely diagnoses (Diagnosis A and B). The experimental intervention was in a "validation" phase preceding the test, where participants were asked to first verify a diagnosis which was either representative of i) Diagnosis A, and similar to a test case, b) Diagnosis A and dissimilar, c) Diagnosis B and similar. Each participant verified 2 cases from each condition, for a total of 6 . Participants were not told of the manipulation. They then diagnosed all 8 test cases (the remaining 2 test cases had no prior). All cases were counterbalanced across conditions. We compared accuracy for cases from Condition a) and b) with the no-prior cases to evaluate the effect of prior exemplar; comparisons between c) and no-prior evaluated the effect of category priming. Because cases were designed so that both Diagnosis A and B were equally likely, overall accuracy was measured as the sum of proportion of cases in which either was selected. Subjects were emergency medicine staff $(\mathrm{n}=40)$, residents $(\mathrm{n}=39)$ and medical students $(\mathrm{n}=32)$ from McMaster University, University of Washington, and Harvard Medical School. Results: Overall, staff had an accuracy $(\mathrm{A}+\mathrm{B})$ of $98 \%$, residents $98 \%$ and students $85 \%(\mathrm{~F}=35.6, \mathrm{p}<.0001)$. For residents and staff there was no effect of condition (accuracy ranged from $97 \%$ to $100 \%$ ); for students there was a clear effect of category priming, with accuracy of $84 \%$ for a), $87 \%$ for b) and $94 \%$ for $\mathrm{d}$ ) but only $73 \%$ for the no prime condition (Interaction $\mathrm{F}=3.54, \mathrm{p}<.002$ )

Conclusion: Although prior research has shown substantial effects of similarity to prior cases, primarily in visual domains, the present study has shown such effects only for medical students. Possible explanations will be explored. Nevertheless, the study shows that with increasing expertise, availability may not be a source of error. 
4.

\section{Patient Safety 4.0: "Mistake of the Week"}

\section{F. F. Ulmer}

Bern University Hospital, University of Bern, Switzerland, Bern, Switzerland

Background: The rate of mistakes and near misses in clinical medicine remains staggering. The propensity to cover-up mistakes thrives in name-blame-shame cultures. The need for recurring safe-zone forums in which mistakes can be discussed openly is evident. Following a comprehensive review of the literature, a semi-structured weekly conference, labeled "mistake of the week" (MOTW), was introduced in late 2014, enabling physicians to voluntarily disclose mistakes and near misses they had made. To minimize the inhibition caused by hierarchy, the two heads of the departments of internal medicine spearheaded the experiment by reporting their mistakes of the preceding week. Over time, attending and resident physicians followed suit and began volunteering mistakes they had made. MOTW conference was introduced with the intention of encouraging cultural change toward how physicians approach, process, accept and learn from their own mistakes. We seek to assess how MOTW is perceived by physicians and medical students of the two departments, what the impact MOTW has on daily routine, which factors were relevant for establishing MOTW and how MOTW could be improved.

Methods: Physicians and medical students of the Medizinische Klinik I and II at the academic teaching hospital Klinikum Konstanz in Germany, at which the MOTW conference was introduced, were eligible to participate voluntarily. Four groups of between three and six physicians and one group of 5 medical students were selected based on their work schedule availability and grouped according hierarchical status (residents, attending physicians, head of the departments), to participate in focus group interviews, facilitated by a moderator using a questioning route. The interviews are videotaped, transcribed and analyzed qualitatively using a thematic analysis approach.

Results: Results of all 5 scheduled focus groups will be presented at the time of the conference. All physicians and medical students, who were contacted, chose to participate. Preliminary results indicate that the MOTW conference enjoys overwhelming acceptance among physicians and medical students alike, irrespective of hierarchical level.

Conclusion: According to our knowledge this is the first report investigating the perception, impact, success factors and lessons learned of an institutionalized medical conference dedicated to disclosing medical mistakes that have occurred. Our preliminary conclusion is that the MOTW conference appears to be an ideal forum to mitigate hierarchy and promote a sustainable organizational dynamic in which mistakes and near misses can be discussed in an environment free from name-blame-shame. 
5.

\title{
The Impact of Disease-Labels on Disease Experience in Patients with Prolonged Incurable Cancer
}

\author{
V. Busink ${ }^{1}$, V. Ho ${ }^{2}$, A. Reyners ${ }^{3}$, E. Smit ${ }^{1}$, H. Buiting ${ }^{1}$, D. Van Tol ${ }^{3}$ \\ ${ }^{1}$ Antoni van Leeuwenhoek Hospital, Amsterdam, Netherlands \\ ${ }^{2}$ Universiteit Utrecht, Utrecht, Netherlands \\ ${ }^{3}$ Universiteit Groningen, Groningen, Netherlands
}

Background: A medical diagnosis can be considered as one of the most important classification tools, as it assigns 'individuals' to the population of 'patients'. Doctors are sometimes selective in the information they receive from patients, which may result in diagnostic oversight or a less than optimal diagnosis. At present, advances in oncology have resulted in prolonged disease trajectories in patients with incurable cancer. This has induced discussions about the 'optimal' medical terminology. We examined the impact of disease-labels on disease experience in patients with prolonged incurable cancer.

Methods: We conducted a qualitative study at the day-care unit of a comprehensive cancer center in the Netherlands. Short conversations with nineteen patients with incurable breast cancer (one year after diagnosis),10 patients with incurable lung cancer (six months after diagnosis), and one patient with ovarian cancer were included, all in stable condition; interviews were analysed following the concepts of thematic analysis. Results: A substantial group of patients $(\mathrm{n}=21)$ used (or explicitly not used) disease-labels in their communication about their disease. They varied in their preference regarding disease-labels (e.g. 'chronic', 'palliative', 'patient'). Patients with a more positive mood seemed more comfortable with the label 'chronic', whereas patients with a less positive mood, seemed to perceive disease-labels such as 'chronic' as a lifelong burden. Some patients preferred not to label their disease at all, as they felt it would make them 'less human'. Healthcare professionals' use of disease-labels could sometimes distress patients, especially when patients heard different disease-labels from different healthcare professionals. Patients, more willing to perceive and label themselves as a 'patient' tended to receive more support. Patients not using any disease-label in their communication $(n=12)$, seemed to be less engaged with their disease and focused more on their personal experience. Conclusion: Patients' use of disease-labels is part of their coping strategy. Given the possible impact of disease-labeling on patient's coping strategies, the importance to communicate appropriate and uniform disease-labels towards patients is clear. More research is warranted to explore which disease-labels suit different patients best and to explore other outcomes than well-being only, such as (the frequency) of treatment decisions. 
6.

\section{Uncovering Clinical Reasoning Processes and Diagnostic Errors of Medical Students in Virtual Patients}

$\underline{\text { I. Hege }}^{1}$, A. A. Kononowicz ${ }^{2}$, L. Foster-Johnson ${ }^{3}$, J. Kiesewetter ${ }^{4}$

${ }^{1}$ Institute for Medical Education, LMU Munich, München, Germany

${ }^{2} J a g i e l l o n i a n$ University Medical College, Krakow, Poland

${ }^{3}$ Geisel School of Medicine at Dartmouth, Hanover, NH

${ }^{4}$ Klinikum der LMU München, Munich, Germany

Background: Clinical reasoning is an important topic in healthcare training, assessment, and research. Virtual patients (VPs) are a safe environment to teach, assess clinical reasoning and diagnostic accuracy. We developed a concept mapping tool, which specifically captures the clinical reasoning process while learning with virtual patients. Our aim was to explore the details of the clinical reasoning process, diagnostic accuracy and errors of medical students when working with VPs using the concept mapping tool to document their reasoning process.

Methods: Over seven months we provided access to 67 German and 30 English VPs in CASUS combined with a concept mapping tool to visualize and measure the clinical reasoning process by identifying problems, differential diagnoses, recommended tests and treatment options, and composing a summary statement about the VP. A final diagnosis had to be submitted by the learners in order to conclude a VP scenario. Learners were allowed multiple attempts or could request the correct diagnosis from the system after one unsuccessful attempt. Errors, such as premature closure or availability bias can be detected by the concept mapping tool.

Results: We analyzed 1,393 completed concept maps from 317 learners. We found significant differences between maps in which learners provided a correct final diagnosis on one (group C) or multiple attempts (group W) and maps in which learners gave up and requested the solution from the system after one or more unsuccessful attempts (group S). Maps in which learners gave up, had lower scores, fewer summary statements, and fewer problems, differential diagnoses, tests, and treatments. A premature closure error occurred significantly more often in group W than in group S; availability biases were not recorded by the tool.

Conclusion: The different use patterns and scores between learners who entered the correct final diagnosis on one or multiple attempts and those who gave up, indicate that diagnostic accuracy in the form of a correct final diagnosis on the first attempt has to be reconsidered as a sole indicator for clinical reasoning competency. The algorithms for detecting cognitive errors are currently expanded to include also base rate neglect, representativeness, confirmation bias, and (non-) availability errors. Further research is needed in this area. For the training, assessment, and research of clinical reasoning skills we suggest focusing more on the details of the process to reach a correct diagnosis, rather than whether it was made in the first attempt. 


\section{Scientific Abstracts}

\section{Poster Session 1 - Scientific Abstracts}

1.

\section{An Audit of Diagnostic Error in All Surgical Deaths in Australia over 7 Years}

D. Pincus $^{1,2}$, T. Rey-Conde ${ }^{3}$, J. North ${ }^{3}$, J. Allen ${ }^{3}$

${ }^{1}$ Leading Steps Paediatric Clinic, Benowa, Australia

${ }^{2}$ Bond University, Gold Coast, Australia

${ }^{3}$ Royal Australasian College of Surgeons, Brisbane, Australia

Background: All patients who die in Australian hospitals under the care of a surgeon are audited. In over $90 \%$ the cause of death was the disease process and no errors are identified. The results of the audit are coded, and used for regular feedback to surgeons and to health authorities for continuous quality improvement. This study looks at the main categories of error and analyses their trends over time, with reference to diagnostic error but also looking at all modifiable factors.

Methods: Data was obtained from six of the seven state Audits of Surgical mortality over the period 2010 to 2016 (the last year full data is available). Remediable causes were clumped into general categories including diagnostic error or delay, surgical error, system errors and errors where either the system or surgeon may have been responsible. Proportions of remediable deaths for each year were ascertained and analysed for trends over time.

Results: Surgical mortality is decreasing with time. Diagnostic error or delay is a small proportion of remediable error in surgical deaths and is constant over the period of the audit. $12.4 \%$ of the remediable deaths were attributed to diagnostic error in 2010 compared with $11.4 \%$ in 2016 . Diagnostic error was relatively more common in some subspecialties. It accounted for 332 or 3\% of 11073 deaths in general surgery, $3.5 \%$ of 171 paediatric surgical deaths, and 5.3\% of 76 deaths in Obstetrics and Gynaecology. Diagnostic error was relatively uncommon in Neurosurgery (1.1\% of 4127 deaths) and orthopaedics ( $0.9 \%$ of 5392 deaths). Surgical error is increasing with the years, particularly error around decision to operate. Of 22,780 deaths during the audited period, $2.15 \%$ had a decision to operate incident in 2010 compared with $3.7 \%$ in 2015 which is strongly statistically significant. System errors tended to decrease with time dropping from $5.4 \%$ to $3.6 \%$ over the same period.

Conclusion: Surgeons hospitals and governments must work together to minimise patient adverse outcomes. Australia has a unique whole of population system to review all surgical deaths. This audit revels that system errors are decreasing, diagnostic error is stable and surgical errors are increasing in importance over a six year period. This is almost certainly related to increasing age, level of illness and co-morbidities amongst patients being surgically managed in Australian hospitals. Efforts to improve surgical mortality should focus on surgical error, particularly decision to operate.

\section{Proportion of patients with diagnostic errors by year}

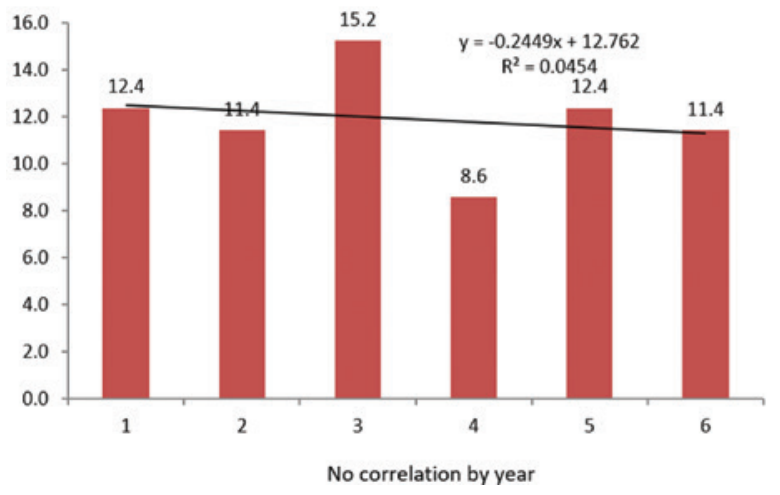


2.

\section{Can We Improve Triage of Chest Pain at out-of-Hours Primary Care By Learning from Fatal Adverse Events? a Case- Control Study}

C. Erkelens, D. L. Zwart, L. Wouters, E. de Groot, R. Damoiseaux, A. Hoes, F. H. Rutten

University Medical Center Utrecht, Utrecht, Netherlands

Background: With a yearly incidence of $0.006 \%$ fatal adverse events or 'calamities' at the primary care out-of-hours service (PC-OHS) are rare. Of PC-OHS calamities, missed diagnosis of acute cardiovascular diseases are most common and flaws in the triage process often are found as root causes. By Dutch law, root cause analysis (RCA) of every single calamity is required, mostly leading to an array of improvement measures for daily practice. However, given the high risk of hindsight bias when outcomes are adverse and the extreme low incidence of calamities, it isquestionable whether the root causes and consequential improvement measures distilled from these rare fatal events will sufficiently add to quality and safety. Moreover, it is unknown whether these calamities are representative for structural flaws in the system. Indeed, it has never been studied whether it is possible at all to distinguish calamities from non-calamities in foresight. With this study we aimed to assess if PC-OHS calls that resulted in calamities differed from matched controls in the domain of patients suspected of an acute coronary syndrome. Methods: We conducted a case-control study at one of the largest PC-OHS in the Netherlands. Triage conversations in the period January 2013- December 2016 that resulted in a cardiovascular calamity were matched 1:4 with other triage conversations based on age, gender and symptoms. A researcher blinded to the outcome, extracted information regarding call (6 items), patient characteristics (22 items) and urgency allocation from the archived phone calls using a standardized case record form. For each call, the researcher tried to predict whether the conversation would end in a calamity or not. Univariable analyses were used.

Results: A total of 14 calamities and 56 matched controls were included. Calamities and controls were very similar. In calamities there was more often an atypical location of the chest pain $(p=0.04)$ and radiation of the pain $(p=0.04)$ than matched non-calamities. Prediction of the eventual outcome resulted in 28,6\% sensitivity (95\% CI 0.10-0.58), 87,5\% specificity (95\% CI 0.75-0.94), 36,4\% positive predictive value ( $95 \%$ CI 0.12-0.68) and 83,1\% negative predictive value (95\% CI 0.71-0.91).

Conclusion: We could not show essential differences in the presentation of calamity and non-calamity calls, which suggests that it is 'impossible' with telephone triage to discover all high urgent cases of patients with chest pain. Hence, prevention of these calamities seems an illusion, and building improvement measures on single calamities therefore questionable. 
3.

\section{Chest Discomfort during Early Morning Hours Means Higher Risk of ACS. an Easy Guidance for Improving Triage in Primary Care out-of-Hours Services}

L. Wouters, D. L. Zwart, C. Erkelens, E. de Groot, R. Damoiseaux, A. Hoes, F. H. Rutten

University Medical Center Utrecht, Utrecht, Netherlands

Background: Early detection of patients with ACS is crucial to initiate early interventions to prevent or reduce myocardial necrosis, and improve outcome and prognosis. In $70 \%$ of patients with chest discomfort who contact primary care out of hours in the Netherlands an ambulance is dispatched. Yet, around $10 \%$ has an acute coronary syndrome (ACS). With just history taking during the initial telephone contact it is difficult to both efficiently and safely triage patients with chest discomfort. There is an urgent need of determinants that are helpful to assist the triagist in her task. It is known there is a peak incidence of ACS in the early morning which can be helpful for urgency allocation during telephone triage. Simple taking the time of calling into account could possibly increase the efficiency of referrals of this acute patient population to the emergency department. The aim of this study was to investigate if patients with chest discomfort calling the PC-OHS between 4am and 8 am have a higher risk of having an ACS than those calling other hours.

Methods: 765 telephone conversations of patients with chest discomfort were re-listened to collect information about patient characteristics, symptoms, medical history and urgency allocation. Final diagnoses were collected at the GP's office. We compared patients calling early morning (4.00-8.00am) with those calling other hours.

Results: In the early morning $24.4 \%$ had an ACS, at other hours $10.0 \%(\mathrm{p}<0.001)$. Early callers were older $(62.9$ years vs. 58.3 , $\mathrm{p}=0.035)$, had more often short lasting chest pain $<12$ hours $(86.3 \%$ vs. $71.0 \%, p=0.006)$, but received similarly high urgency allocation $70.9 \%$ vs. $65.5 \%$ $(\mathrm{p}=0.319)$

Conclusion: Patients with chest discomfort calling the PC-OHS between 4am and 8am have a more than twice a higher risk of ACS than those calling other hours. Ambulances may be dispatched 'without restriction' in these hours for these patients. 
4.

\section{Clinical Diagnostic Error Rates in a Outpatient Dermatology Adult Clinic}

\section{J. Wu${ }^{1}$, E. J. Lowenstein ${ }^{2}$}

${ }^{1}$ suny health science center brooklyn, brooklyn, NY

${ }^{2}$ suny health science center brooklyn, valley stream, NY

Background: In dermatology, data regarding diagnostic error remains limited. We designed a study to assess accuracy in predicting clinical dermatologic diagnoses.

Methods: An IRB exempt, retrospective review of 230 adult patient charts at King's County Hospital Dermatology Clinic was conducted from patients seen between April and September 2016. The study was designed to assess the rate of diagnostic discordance between clinical and histologic diagnoses (our "gold standard"). A "correct" diagnosis was deemed if any one of the clinical differentials was consistent with the final histopathologic diagnosis, as read by a board-certified dermatopathologist. Of the discordant diagnoses, we further quantified how many were clinically significant or required a different treatment approach.

Results: Data review showed an overall 20\% discordance rate. Within the 4 diagnostic classes, inflammatory/reactive (27\%) and neoplastic (24\%) diagnoses harbored higher discordant rates than autoimmune (0\%) and infectious (3\%) etiologies. Of the discordant inflammatory/ reactive cases, $77 \%$ required consideration of a potentially different treatment. Of the discordant neoplastic cases, only $2(12.5 \%)$ were clinically significant. The rate of diagnostic discordance was similar between cases where a differential diagnosis was listed (22\%) and not (19\%). Conclusion: This study suggests higher diagnostic accuracy for autoimmune (discordant rate of $0 \%$ ) and infectious dermatoses (discordant rate of 3\%) by clinical assessment alone. Inflammatory/reactive cases showed the greatest discordant rate (29\%), which we may speculate is a consequence of less specificity in clinical presentations. These finding highlight the importance of clinical correlation with histology in clarifying inflammatory and neoplastic disorders. The similar rates of discordance between cases where a differential was listed and not suggests that expressing a differential diagnosis did not imply less diagnostic confidence. 
5.

\section{Diagnostic Error Increases Mortality and Length of Hospital Stay in Patients Presenting through the Emergency Room: A Prospective Observational Study}

S. C. Hautz ${ }^{1}$, J. E. Kämmer², T. C. Sauter ${ }^{1}$, L. Zwaan ${ }^{3}$, S. K. Schauber ${ }^{4}$, A. K. Exadaktylos ${ }^{1}$, T. Birrenbach ${ }^{1}$, V. Maier ${ }^{5}$, W. E. Hautz ${ }^{1}$

${ }^{1}$ Inselspital, Bern University Hospital, Bern, Switzerland

${ }^{2}$ Charité Universitätsmedizin Berlin, Berlin, Germany

${ }^{3}$ Erasmus MC, Rotterdam, Netherlands

${ }^{4}$ University of Oslo, Oslo, Norway

${ }^{5}$ Spital Solothurn, Solothurn, Switzerland

Background: Diagnostic errors are frequent and have severe consequences. Most studies on this topic, however, analyze cases of error only, making it difficult to identify case characteristics unique to diagnostic error. Our objective was to determine the rate of diagnostic error in patients hospitalized through the emergency room, identify factors predicting such errors, and their consequences.

Methods: We collected data through a prospective observational study in one university-affiliated tertiary care hospital. Patients' hospital discharge diagnosis was compared with the diagnosis at hospital admittance through the emergency room and classified as similar or different according to a predefined scheme by two independent expert raters. A generalized linear mixed-effects model was used to determine whether characteristics of patients, diagnosing physicians, and context predicted diagnostic error. We further assessed in-hospital mortality, length of hospital stay, and diagnostic error, defined as discrepancy between primary admittance and discharge diagnoses.

Results: 755 consecutive patients were included, diagnostic error identified in 12.3\% of cases. Diagnostic error was associated with a longer hospital stay (mean 10.29 vs. 6.90 days; $\mathrm{P}=0.038$; odds ratio 1.34; $95 \%$ confidence interval 1.02 to 1.76 ) and increased patient mortality (8 $(8.60 \%)$ vs. $25(3.78 \%) ; \mathrm{P}=0.007$; odds ratio $3.94 ; 1.46$ to 10.60$)$ as compared to no error. A factor available at admittance that predicted diagnostic error was the diagnosing physician's assessment that the patient presented atypically for the diagnosis assigned ( $\mathrm{P}<0.001$; odds ratio $2.71 ; 1.51$ to 4.86 ).

Conclusions: Discrepancies between the emergency room admittance diagnosis and the hospital discharge diagnosis occur in every ninth patient and are associated with increased in-hospital mortality. Diagnostic errors are not readily predictable by fixed patient or physician characteristics but seem to depend on context. 
6.

\title{
Diagnostic Errors in Respiratory Medicine: A Possible Method of Assessment
}

\author{
$\underline{\text { R. A. Crisan-Dabija }}{ }^{1,2}$, T. Mihaescu ${ }^{3}$ \\ ${ }^{1}$ Clinic of Pulmonary Diseases, Iasi, Romania \\ ${ }^{2}$ University Of Medicine and Pharmacy “Grigore T. Popa” Iasi, Iasi, Romania \\ 3”Grigore T. Popa” University of Medicine and Pharmacy, Iasi, Romania
}

Background: The diagnostic errors are a controversial, hot-topic but also a real-life concern. Starting with 1999 Institute of Medicine's publication: “To Err is Human”, academic and mass-media's interest grew regarding unintended harm due to medical-care procedures. The alarming figures stating that 1 in 10 patients may be harmed due to medical error may be the result of overwhelmed physicians in their attempt to optimize the diagnosis and treatment process.

Methods: Using a database analysis of patients admitted and released from our Clinic of Pulmonary Diseases, we proposed a formula to assess the possibility to identify the diagnostic errors since Romania lacks connected nation-wide medical records. We analyzed data of the patients admitted, released and deceased within a 35 months' period (23789 entries), between January 1st, 2015 - December $31^{\text {st }} 2017$. Database was constructed using patients' identification data, ICD-10-CM diagnostic codes, diagnostic on admittance, diagnostic after $72 \mathrm{~h}$, diagnostic on release (or if decease, diagnostic based on necropsy). To assess possible red-flags on diagnostic error, we used the following triggers: correspondence between admittance diagnostic and release diagnostic, re-admittance earlier than 30 days with same or other diagnostic, admittance diagnostic and necropsy diagnostic. Based on this data, we constructed a score-card, attributing "Score =0" for correspondence between admittance and release or necropsy diagnostic, "Score $=1$ " for non-correspondence between admittance and release diagnostic (primary or any other first 3 secondary diagnostics), "Score =2" for non-correspondence between admittance and necropsy diagnostic, "Score =3" for readmittance earlier than 30 Days with other diagnostic and "Score=4" for readmittance earlier than 30 Days and non-correspondence between admittance and necropsy diagnostic (Figure1).

Results: 33,9\% (9212) of patients had no correspondence between admittance and release diagnostic (Score=1). 7,18\% (1708) had different cause of death than the admittance diagnostic (Score =2), 5\% (1202) of patients returned earlier than 30 days after release (Score $=3$ ) and 1,8\% of patients were admitted earlier than 30 days after release, with other diagnostic of admittance and non-concordance between admittance and necropsy diagnostic, hence receiving a Score $=4$ (431). Since scores of 1 and 2 may be considered possible errors in diagnostic process, we considered patients framed in Score 3 and 4 as probable diagnostic errors.

Conclusion: Diagnostic errors are difficult to asses and more difficult to be accepted by the physicians, hospital officials and patients. The proposed formula may be a way to make these errors quantifiable and opens a possible window to an attempt to prevent them.

\begin{tabular}{|c|c|}
\hline $\begin{array}{l}\text { Score }=1 \\
\text { non-correspondence } \\
\text { between admittance and } \\
\text { release diagnostic }\end{array}$ & $\begin{array}{l}\text { Score }=2 \\
\text { non-correspondence } \\
\text { between admittance } \\
\text { and necropsy diagnostic }\end{array}$ \\
\hline $\begin{array}{l}\text { Score }=3 \\
\text { readmittance earlier than } \\
30 \text { Days with other } \\
\text { diagnostic }\end{array}$ & $\begin{array}{l}\text { Score }=4 \\
\text { non-correspondence } \\
\text { between re- } \\
\text { admittance (earlier } \\
\text { than } 30 \text { days) and } \\
\text { necropsy diagnostic }\end{array}$ \\
\hline
\end{tabular}


7.

\section{Failed Communication of New Diagnoses and Treatment Plans in Hospital to the Primary Care Physician Identified Both in Medical Records and a Patient Survey}

M. Van Melle Jr. ${ }^{1}$, J. Poldervaart Jr. ${ }^{2}$, H. van Stel Sr. ${ }^{2}$, N. de Wit Sr. ${ }^{2}$, D. L. Zwart ${ }^{3}$

${ }^{1}$ University of Cambridge, Cambridge, United Kingdom

2UMC Utrecht, Julius Centre, Utrecht, Netherlands

${ }^{3}$ University Medical Center Utrecht, Utrecht, Netherlands

Background: Referrals form a critical part of the diagnostic pathway. Between-provider communication of newly established or excluded diagnoses of referred patients is however often problematic. The aim of this study is to assess the communication rate of new diagnoses and associated treatment plans found in hospital to the primary care physician (PCP).

Methods: This descriptive study (2015-2016) was part of a broader study on incident prevention during patient transitions (TIPP). Concordant with the emphasis on patient communication included in the IOM 2015 definition of diagnostic errors, we studied both linked (1ry/2ry care) medical records and patient-reported data. For the medical records, we studied patient referrals to the cardiology or gastroenterology department in two hospitals in the Utrecht region. For the survey, we included patient referrals to the cardiology and gastroenterology department in two regions in the Netherlands (Utrecht and Hardenberg). In the medical records, we identified delayed (received $>14$ days after diagnosis) and missing correspondence to the PCP, and assessed if new diagnosis and associated treatment plan were registered in the PCP medical record. In a validated patient reported experience survey (the Transitional Risk and Incident Questionnaire = TRIQ) we scored items indicating failing communication.

Results: In the linked medical records of 395 referred patients ( 354 to the outpatient clinic, 41 to the A\&E), correspondence letters of 112 (28.4\%) patients were missing and of $99(25 \%)$ patients were delayed. Of 197 new diagnoses identified in the hospital record, 13 (6.6\%) were not registered in the PCP record and 44 (22.3\%) were registered inadequately. Using the TRIQ, we surveyed 178 recently referred patients. Of the 86 patients with a new diagnosis and/or treatment plan, 9 (10.4\%) reported that their PCP had not been informed.

Conclusion: We demonstrate that diagnostic referrals are often associated with failed communication of new diagnoses and treatment plans from hospital to the PCP. These alarmingly frequent events increase the risk of serious diagnostic safety incidents. Interventions to provide fail-safe mechanisms and informational integration of the diagnostic process need to be developed and evaluated. 
8.

Heuristic in a Dermatologic Approach to Skin Disease

E. J. Lowenstein

suny health science center brooklyn, valley stream, NY

Background: Little has been published on how we make diagnoses in dermatology, where we err and how we can improve our diagnoses. Based on previous findings in cognitive research, flawed thinking and misinterpretation of visual findings are the most likely cause of most diagnostic error in dermatology.

Methods: This talk focuses on heuristics used specifically in dermatologic approach to disease, with clinical examples given to demonstrate how a heuristic approach is helpful but needs to be monitored for cognitive and visual traps.

Results: Examples are given of the classic eight diagnostic heuristics of dermatology: Primary lesion, morphology/shape, location, distribution, pattern, color, feel/texture and context.

Conclusion: A better understanding of how a dermatologist traditionally approaches disease can help physicians avert some predictable diagnostic error. 
9.

\section{Higher Risk of Emergency Colon Cancer Diagnosis in Women after a Recent Benign Diagnosis: A Data-Linkage Study in England on Pre-Diagnostic History}

C. Renzi ${ }^{1}$, Y. Lyratzopoulos ${ }^{1}$, W. Hamilton ${ }^{2}$, B. Rachet ${ }^{3}$

${ }^{1}$ University College London, London, United Kingdom

${ }^{2}$ University of Exeter, Exeter, United Kingdom

${ }^{3}$ LSHTM, London, United Kingdom

Background: Emergency diagnosis of colon cancer is associated with worse survival. To identify opportunities for earlier diagnosis and reducing emergency presentations for cancer, we evaluated consultation patterns, symptoms and benign diagnoses recorded before emergency and non-emergency presentations.

Methods: Cohort study on 5,745 colon cancer patients diagnosed in England 2005-2010, with individually-linked cancer registry, primary and secondary care data for the 5-year pre-diagnostic period.

Results: Colon cancer was diagnosed as an emergency in 34\% of women and 30\% of men. Primary care consultations with relevant symptoms increased markedly during the pre-diagnostic year among emergency and non-emergency presenters. Among emergency presenters, $20 \%$ of women and $15 \%$ of men $(\mathrm{p}=0.002)$ had alarm symptoms (anaemia, rectal bleeding, change in bowel habit) 2-12 months pre-diagnosis. Women with abdominal symptoms (change in bowel habit/constipation/diarrhoea) received a benign diagnosis (irritable bowel syndrome (IBS)/ diverticular disease) more frequently than men in the pre-diagnostic year: $12 \%$ versus $6 \%$ among women and men diagnosed as emergencies $(\mathrm{p}=0.002)$. Emergency diagnosis was more likely in women $(\mathrm{OR}=1.20 ; 95 \% \mathrm{CI} 1.1-1.4)$, independently of cancer sub-site. Benign diagnoses in the pre-diagnostic year $(\mathrm{OR}=2.01 ; 95 \% \mathrm{CI} 1.2-3.3)$ and anaemia 2-5 years pre-diagnosis $(\mathrm{OR}=1.91 ; 95 \% \mathrm{CI} 1.2-3.0)$ increased the risk of emergency presentations in women but not in men. The risk was particularly high for women aged 40-59 with a recent benign diagnosis versus none (OR=4.41; 95\%CI 1.3-14.9).

Conclusion: Colon cancer should be carefully considered in women aged 40-59 with symptoms consistent with a recent onset of IBS or diverticular disease. One-fifth of women diagnosed as emergencies had alarm symptoms in the pre-diagnostic year offering opportunities for earlier diagnosis. 
10.

\section{Impact of Audit-Feedback on the Test Threshold for CT Pulmonary Angiogram}

\section{T. K. LIM, J. Zhang}

National University Hospital, Singapore, Singapore

Background: A low test threshold for pulmonary embolism (PE) and over reliance on imaging, especially CT pulmonary angiograms (CTPA) is a highly prevalent behaviour among physicians in acute medicine. Interventions for this diagnostic base-rate error have been mostly ineffective. Methods: This is a prospective study of consecutive hospitalized patients who underwent CTPA in an acute medicine department. We implemented an audit-feedback program of increasing intensity to improve the diagnosis of pulmonary embolism from CTPA in hospitalized patients. In 2016 we conducted physician education and periodic collective feedback. This was supplemented, in 2017, by a more stringent PE testing protocol and, in collaboration with the radiology department, real-time feedback for compliance with explicit pre-test risk estimates and age-adjusted D-dimer levels.

Results: On monitoring long term trends we detected a fall in PE diagnosis with CTPA, from $\sim 15 \%$ to below $10 \%$ over a decade of increasing accessibility to advanced imaging. In 2015 this rate was 6.7\%. In 2016, with low intensity feedback, it was 7.8\%. In 2017, the higher intensity feedback was associated with more frequent assessment of pre-test risks and D-dimer testing. There was a small increase in PE diagnosis from CTPA to 11.3\% (not significant). In 2018 we have implemented a modified version of the Dutch YEARS protocol (Lancet 2017; 390:289). Conclusion: Physician education and real-time feedback had a small effect in improving adherence to PE diagnostic protocols but negligible effect on base-rate errors in CTPA testing. In this regard, the innovative Dutch YEARS protocol needs further evaluation in secular practice. 
11.

\title{
In the Blink of an Eye: Rapid Visual Diagnosis
}

\author{
S. Monteiro, J. Sherbino, G. Norman, M. Sibbald \\ McMaster University, Hamilton, ON, Canada
}

Background: Within a competency based medical education era is a need to develop a new set of assessment instruments that determine competent practice in an authentic environment. While many skills must be evaluated in a context or specialty specific manner, visual diagnosis is a complex task nearly universal to medicine. X-rays and (often ECGs) are part of internal medicine, surgery emergency medicine, pediatrics, radiology and related subspecialties. The goal of the current study was to develop an assessment tool that establishes a benchmark of performance for diagnosing ECGs and lung radiographs.

Methods: Using a speeded protocol in which participants view diagnostic images for very brief time windows, we tested physicians' sensitivity to detecting abnormalities in ECGs and lung radiographs. This protocol has been shown to discriminate between novices and experts when diagnosing mammograms, however this is the first attempt to use this protocol as a competency assessment tool. We consulted experts from Radiology and Cardiology to develop materials for each specialty/format of imaging. Residents (12) and staff physicians (17) in Emergency Medicine were recruited as participants. Each participant completed two versions of the study. In both versions participants viewed diagnostic images and indicated whether the image was clinically normal or abnormal. Version 1 contained 100 ECGs and version 2 contained 100 lung radiographs. In each version, 50 images were normal and 50 were abnormal. Images were displayed at one of four time windows: $175 \mathrm{~ms}, 250 \mathrm{~ms}, 500 \mathrm{~ms}$ and $1000 \mathrm{~ms}$. Images and timing windows were counterbalanced to ensure a balanced design. For each participant, we calculated a d' statistic as a threshold independent, normally distributed measure of accuracy, at each exposure time.

Results: Average d' was 0.83 for ECGs and 0.97 for radiographs. Critically, d' for junior residents was lower, (0.95) than senior staff (1.12) for radiographs. Similarly, d' for junior staff and residents was lower (0.79) than senior staff (0.87). As shown in previous studies, d' was smallest for the shortest viewing time, on average (0.72) at $175 \mathrm{~ms}$ compared to (1.10) for $1000 \mathrm{~ms}$ for both versions.

Conclusion: The current study demonstrates the potential for using speeded tests as indicators of experience and competence. While indepth data gathering and additional diagnostic testing may help confirm a working diagnosis, physicians rely only on their own experience to determine if there is any underlying pathology present in a diagnostic image. The study is a novel approach to isolating the effect of direct experience on clinical expertise. 
12.

\section{Learning to Diagnose X-Rays: Seeing Many Examples Is More Effective Than Receiving Extensive Feedback on Few Examples}

L. Zwaan ${ }^{1}$, B. Hussain ${ }^{1}$, A. Devos ${ }^{1}$, W. W. Van den Broek ${ }^{1}$, S. Mamede ${ }^{1}$, H. Schmidt ${ }^{2}$

${ }^{1}$ Erasmus MC, Rotterdam, Netherlands

${ }^{2}$ Erasmus University, Rotterdam, Netherlands

Background: Improving the diagnostic skills of medical students is considered as an effective way to reduce diagnostic errors. Many educational strategies have been studied to improve students' ability to distinguish one disease from another. However, the one strategy that distinguishes experienced and inexperienced physicians -seeing many patients- has not been applied in medical education. In the undergraduate curriculum, generally only few typical examples of diseases are discussed. This study examines whether it would be more effective to practice with many cases while receiving little feedback, compared to few cases with extensive feedback.

Methods: We set up a two-phased experimental study. To study the diagnostic process in a realistic setting while allowing to diagnose many cases, we studied X-ray diagnosis. Two hundred twenty-one first-year medical students were randomly assigned to one of the two conditions (i.e. practice with 46 images with little feedback or 8 images with extensive feedback). See Figure 1. After an introduction, participants practiced with diagnosing two similar looking abnormalities (i.e. either atelectasis versus pleural effusion, or pneumothorax versus skin folds) and images without abnormalities. One week later all participants diagnosed 50 images of abnormalities they practiced with and abnormalities they did not practice with. Additionally, the amount of time they needed to diagnose the images was recorded.

Results: The students who practiced with many images with little feedback performed better in the test phase than the participants who had diagnosed few images with extensive feedback $(p<0.05)$. Additionally, the students who practiced with many cases were significantly faster to diagnose the images in the test phase than the participants who practiced with few cases $(\mathrm{p}<0.001)$. There was an overall learning effect regardless of experimental condition, thus students performed better on the abnormalities they practiced with compared to the abnormalities that had not been exposed to in the learning phase $(\mathrm{p}<0.001)$.

Conclusion: Being exposed to many cases and therefore many different presentations of abnormalities leads to better performance on new cases of the same abnormality as well as a faster diagnostic process. Exposing medical students to more cases early in medical school may be an effective way to improve their diagnostic skills. Further research is required to show the effect at different levels of expertise and for a larger variety of diseases in the educational module.

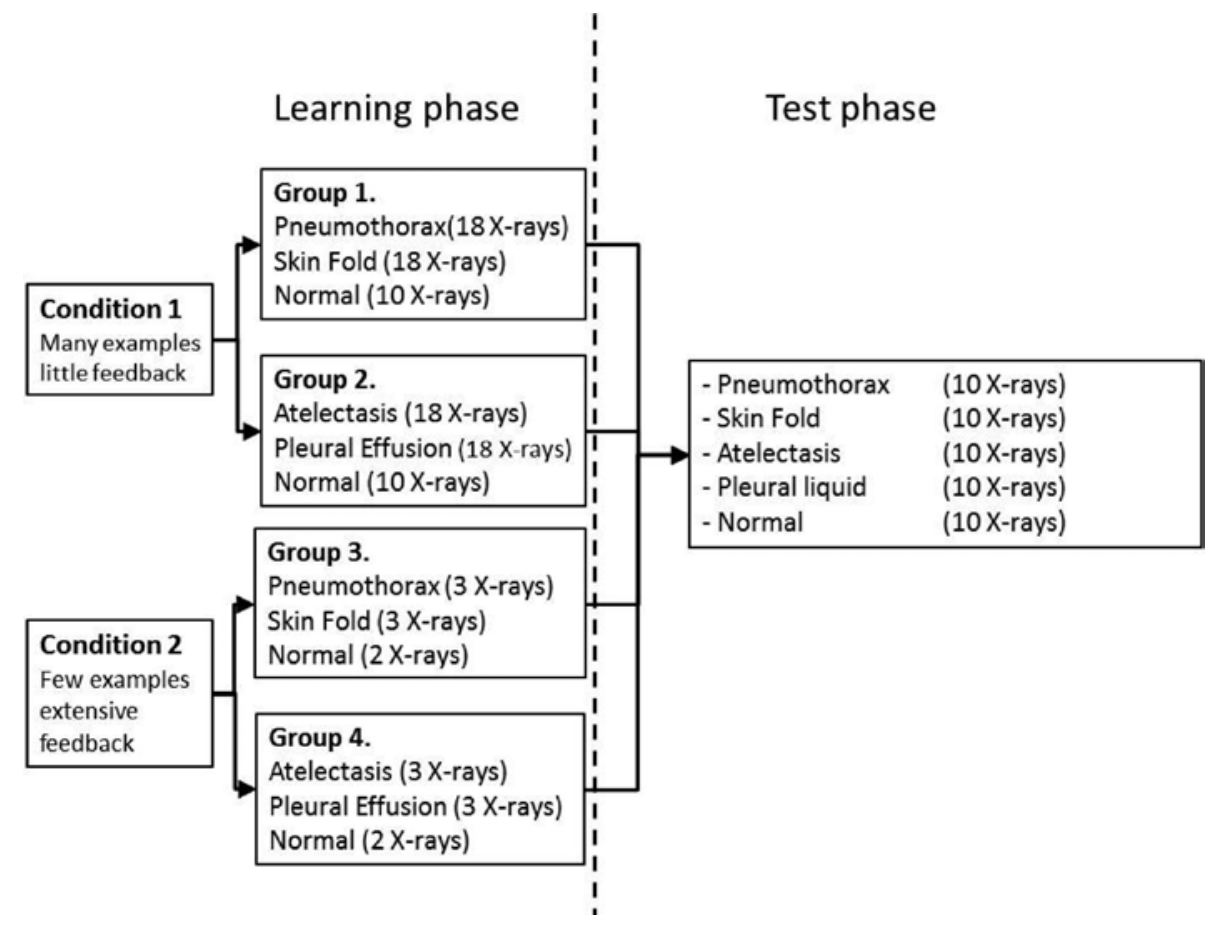


13.

\section{Medical Students` Diagnostic Errors: A Classification}

$\underline{\text { L. T. Braun }}^{1}$, L. Zwaan², K. F. Borrmann' ${ }^{1}$ J. Kiesewetter ${ }^{3}$, M. R. Fischer ${ }^{4}$, R. Schmidmaier ${ }^{5}$

${ }^{1}$ Klinikum der LMU, Munich, Germany

2Erasmus MC, Rotterdam, Netherlands

${ }^{3}$ Klinikum der LMU München, Munich, Germany

${ }^{4}$ Institute for Medical Education, Munich, Germany

${ }^{5}$ Ludwig-Maximilians-University Munich, Munich, Germany

Background: Many studies have focused on diagnostic errors made by physicians, nevertheless it is still unclear which cause leads to the most errors in a diagnostic process. Methodical, it is difficult to classify diagnostic errors correctly due to hindsight bias. To understand the origin of these errors more comprehensively, analyzing diagnostic errors made by medical students could be useful. However, little is known, why students' diagnostic process leads to a wrong diagnosis.

Methods: In two studies, June 2016 and June 2017, 236 medical students worked on eight/fifteen clinical cases in internal medicine. After each clinical case, students wrote down their final diagnosis and an explanation, why they came to their diagnostic conclusion. 1118 explanations were qualitatively analyzed. Furthermore, the diagnostic steps, especially the data gathering process, was tracked and assessed.

Results: There are eight different causes for diagnostic errors: a lack of skills, a lack of knowledge, premature closure, faulty context generation, over/underestimating, misidentification, faulty triggering and cluelessness. The error-distribution is greatly depended on the content of a clinical case. Different cases provoke different kinds of errors. All in all, a lack of diagnostic skills (e.g. interpretation of an electrocardiogram) and an inadequate knowledge base were the main reasons for diagnostic errors.

\begin{tabular}{lr}
\hline Error type & Percentage (number of errors/1118 misdiagnoses) \\
\hline Skills inadequate & $21 \%(234)$ \\
Knowledge inadequate & $18 \%(196)$ \\
Premature closure & $15 \%(170)$ \\
Faulty context generation & $12 \%(139)$ \\
Over/underestimating & $12 \%(136)$ \\
misidentification & $12 \%(133)$ \\
Faulty triggering & $6 \%(69)$ \\
Cluelessness & $4 \%(42)$ \\
\hline
\end{tabular}

Conclusion: The causes for errors made by medical students are comparable to those made by physicians. A lack of diagnostic skills is an important cause for misdiagnoses - the findings are valuable for improving medical education. Furthermore, our method to classify diagnostic errors turned out to be valuable as the diagnostic process remained uninterrupted and hindsight-bias was avoided. 
14.

\section{Non-Specific Complaints at Emergency Department Presentation Result in Low-Quality Diagnoses, Lengthened Hospitalization and Increased in-Hospital Mortality: A Prospective Observational Study}

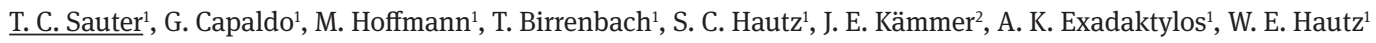

${ }^{1}$ Inselspital, Bern University Hospital, Bern, Switzerland

${ }^{2}$ Charité Universitätsmedizin Berlin, Berlin, Germany

Background: Up to $20 \%$ of patients admitted to an emergency department (ED) present with non-specific complaints (NSC). Retrospective studies suggest an increased risk of misdiagnosis and hospital admission for these patients, but prospective comparisons of the outcomes of ED patients with non-specific complaints (NSC) vs. specific complaints (SC) are lacking.

Methods: All consecutive patients $\geq 18$ years of age admitted to any internal medicine ward at Bern University Hospital via the ED from August 15th 2015 to December $7^{\text {th }} 2015$ were prospectively included and followed up upon. Patients with NSC were compared against those with SC regarding the quality of their ED diagnosis, length of hospital stay and in-hospital mortality.

Results: 711 patients, 165 (23.21\%) with NSC and 546 with SC, were included in this study. No differences between patient groups regarding age, gender or initial severity of the medical problem (deducted from triage category and treatment in a resuscitation bay) were found. Patients with NSC received more unspecific diagnoses (30.3\% vs. 23.1\%, p=0.039, OR $=1.44$ [95\% CI $0.98-2.14]$ ), were hospitalized significantly longer $(\mathrm{Mean}=8.57(\mathrm{SD}=8.74)$ vs. $7.18(6.28)$ days, $\mathrm{p}=0.012, \mathrm{~d}=0.2)$ and had a higher mortality than patients with $\mathrm{SC}(7.3 \%$ vs. $3.7 \%$, $\mathrm{p}=0.045$, OR $2.06[95 \%$ CI $1.01-4.32])$.

Conclusions: Patients presenting with NSC to an ED have an increased risk of an unfavorable outcome, including a doubled risk of in-hospital mortality, despite being otherwise comparable to patients with specific complaints. 
15.

\title{
Novel Approaches to Reducing Diagnostic Errors in Future Doctors: Feasibility Trial of the Electronic Clinical Reasoning Skills Educational Simulation Tool
}

\author{
R. Plackett ${ }^{1}$, J. Sheringham 1 , A. P. Kassianos ${ }^{1}$, S. Duffy ${ }^{2}$, R. Raine ${ }^{1}$ \\ ${ }^{1}$ UCL, London, United Kingdom \\ ${ }^{2}$ Queen Mary University of London, London, United Kingdom
}

Background: Over 80\% of cases of diagnostic errors result in some degree of harm to patients. The leading cause of diagnostic errors are flaws in doctors' clinical reasoning - thought processes required to reach clinical decisions. It is therefore recommended that training on clinical reasoning skills should be integrated into medical education. Online patient simulations could help students to practise such skills in a safe environment. However, there are gaps in our understanding of their impact on clinical reasoning. The aims of this research were to evaluate the feasibility and potential impact of using online patient simulations to improve clinical reasoning in future doctors.

Methods: eCREST (electronic Clinical Reasoning Skills Educational Simulation Tool) seeks to address cognitive biases that can impair clinical reasoning, using interactive 'patient' cases. Informed by iterative hypothesis testing, eCREST prompts students to continually justify their diagnoses. A randomised feasibility trial in two UK medical schools was undertaken. After randomisation, the intervention group received three online patient simulations, the control received teaching as usual. After one week, all students completed a clinical reasoning survey. One month after baseline, all students completed a further case and reasoning survey. A linear and logistic regression were performed to assess the impact on clinical reasoning after one month.

Results: 248 final-year students tested eCREST; 128 were randomised to the intervention. One week after baseline, 72\% (92/128) of the intervention group and $67 \%$ (80/120) of controls remained in the study. One month after baseline, 55\% (71/128) of intervention students and 49\% (59/120) of controls completed follow-up. Of the intervention group, 80\% (73/91) agreed that they learned clinical reasoning skills applicable to clinical work. One month after baseline, the intervention group were significantly more likely to gather essential information from the 'patient' than controls (Odds Ratio $=1.6$; 95\% CI 1.2-2.1) and had a non-significant increase in flexibility in thinking (Mean $=84.1 \mathrm{vs} 81.8, p=0.4$ ).

Conclusion: This feasibility trial provides the necessary evidence to support a larger trial to establish how to integrate eCREST into different curricula and to test its effectiveness. Impact data suggested eCREST improved the way students approached reaching a diagnosis. Simulation tools like eCREST offer a potentially unique approach to teaching clinical reasoning skills. They allow students to pause and reflect on reasoning in real-time, with realistic patient cases. Developing reflective practice skills at an early stage of medical education may contribute to reducing future diagnostic errors. 
16.

\title{
Physicians’ Perceptions of Inappropriate Laboratory Testing in Clinically Admitted Patients
}

\author{
B. Vrijsen, M. ten Berg, C. Naaktgeboren, L. Vos, W. van Solinge, K. Kaasjager
}

UMC Utrecht, Utrecht, Netherlands

Background: Overtesting in laboratory medicine is common. We set up this study to ascertain the appropriateness of laboratory test ordering in admitted patients and to investigate staff's perceptions of, attitudes towards and reasons for the inappropriateness.

Methods: Information was collected on all laboratory tests ordered for patients admitted to the internal medicine ward of our hospital, a large university medical center, from January 2011 to December 2016. Several prespecified measures of inappropriate testing were assessed using these data including the number of fixed combinations of tests, the rate of repetition of laboratory test results within normal range, the number of laboratory test orders per week, and the time to repetition of CRP measurement and its clinical consequences. A survey was performed to investigate perceptions of and attitudes towards the appropriateness. The results of this survey were used as starting point in a series of focus group discussions.

Results: In $97 \%$ of orders that included an ALT, AST was also measured. For sodium and potassium, thus occurred in 95\% of orders. Laboratory test results within the normal range are repeated in up to $85 \%$ of cases. Admitted patients on average have 5.7 laboratory test orders per week. In 59\% of patients in whom a CRP was measured in the Emergency Department directly prior to admittance, CRP was repeated within 24 hours. In $88 \%$ of cases, this did not lead to changes in patient management. In the survey, respondents' estimates of the number of laboratory test orders per week are substantially lower than the actual number (3.9 vs. 5.7). Nevertheless, the majority of respondents think the number of laboratory test orders is too high. Most point to the residents as being most responsible for ordering laboratory tests. In focus groups the causes of excessive laboratory test ordering were explored. Most prominently, the residents felt they did not receive enough feedback. At the same time they considered laboratory test ordering to be too trivial to ask for more feedback from their supervisors.

Conclusion: Inappropriate laboratory test ordering is common and underestimated.. Residents working on the wards are considered to be most responsible for inappropriate laboratory test orders. They feel they need more feedback to change their behaviour, yet think the subject is too trivial to ask for feedback themselves. This implies that interventions to reduce inappropriate laboratory test ordering should focus on facilitating feedback. 
17.

\title{
Prevalence of Diagnostic Error in Referred Outpatients with Fever of Unknown Origin
}

\author{
Y. Harada, T. Hirosawa, K. Morinaga, H. Takase, M. Nin, T. Shimizu \\ Dokkyo Medical University Hospital, Shimotsuga, Japan
}

Background: Fever is one of the most common symptoms when patients present to clinicians, and correctly diagnosing the cause of fever of unknown origin (FUO) remains challenging. Diagnostic error appears to occur frequently in patients with FUO; however, there is a paucity of data regarding the prevalence of such error in patients with FUO, particularly in referred outpatients. We conducted the current study to evaluate the prevalence of diagnostic error in referred outpatients with FUO and to evaluate the factors associated with such error.

Methods: Patients who were referred between October 2016 and September 2017 to the outpatient department of Diagnostic Medicine in a university hospital (from other outpatient departments or different clinics and hospitals) for further evaluation of the cause of FUO were retrospectively included in the present study. The exclusion criteria were as follows: patients with recurrent fever, patients who were admitted prior to diagnosis, or patients whose fever improved before the referral visit. Patients were categorised into either diagnostic error (wrong, delayed, or missed diagnosis) or non-error groups. Differences in clinical characteristics between patients with and without diagnostic error were assessed using the Wilcoxon rank sum test for continuous variables and the chi-square test for categorical variables. A multivariate logistic regression model was used to identify the independent variables of diagnostic error.

Results: In total, 55 patients were included in this study; 29 males and 26 females. The median age was 42.0 years, median duration of fever from onset to referral visit was 1 week, median office visits before referral was 3, and antibiotics were prescribed prior to referral in 30 cases. Diagnostic error was detected in 40 patients $(72.7 \%)$. Patients with diagnostic error visited the clinicians significantly more often before referral compared to those without diagnostic error (median, 3 vs. $1, p=0.01$ ). Moreover, in the multivariate logistic regression model, the number of office visits prior to referral was independently associated with diagnostic error (odds ratio $=2.54$ for 1 time increase; $95 \%$ CI, $1.38-5.60$; $p<0.01)$

Conclusion: In referred outpatients, diagnostic error was detected in almost $75 \%$ of patients with FUO. Frequent office visits prior to referral were associated with the risk of diagnostic error related to FUO. 
18.

\title{
Processes and Outcomes of Negotiating Diagnostic Uncertainty in Specialist Stroke Centres in the United Kingdom
}

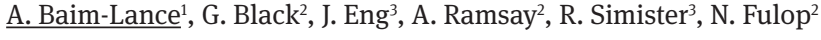 \\ ${ }^{1}$ City University of New York Graduate School of Public Health and Health Policy, New York, NY \\ ${ }^{2}$ University College London, London, United Kingdom \\ ${ }^{3}$ University College London Hospital, London, United Kingdom
}

Background: Hyperacute Stroke Units (HASUs) in Greater London provide comprehensive, around the clock access to urgent stroke care including emergency department thrombolysis, and ward-based multi-disciplinary care from physicians, nurse specialists, and occupational and physical therapists. These services are beneficial because timely intravenous thrombolysis combined with appropriate care and treatment drastically improves stroke outcomes. However, stroke is difficult to diagnose due to confounding symptoms with other conditions such as drugs or alcohol use, systemic infections, tumors, and toxic-metabolic disturbances. These so-called 'mimic' conditions may constitute as much as one-third of hospital presentations. Medical and nursing staff must rapidly negotiate diagnostic uncertainty to reduce medical error and perform timely scans, tests and interventions. In this qualitative research study, we examine how diagnostic uncertainty is experienced and negotiated by medical staff in London's HASUs, the organizational factors driving uncertainty management, and the effects on patients' care pathways.

Methods: We conducted 76 semi-structured interviews with HASU staff (medical, nursing, therapy, administrative and managers), 31 interviews with stroke patients and carers, and 41 non-participant observations of HASU activity conducted four times at each site during weekdays and weekends. Topic and template guides were used for data collection during interviews and observations. Inductive and deductive thematic analysis were used.

Results: There are three crucial time points at which diagnostic uncertainty is negotiated: symptom onset, arrival at accident and emergency, and the decision to admit patients to the HASU ward. One overarching issue is the attraction of the centralized HASU to manage suspected strokes, thereby resulting in stroke teams' rapid management of a wide berth of suspected cases. While thrombolysis appears to be appropriately delivered, a greater issue is over-admitting those with an initial incorrect or uncertain stroke diagnosis to the HASU ward with resulting consequences on quality of care, staff activities, and patient experiences once a non-stroke diagnosis is later confirmed.

Conclusion: Uncertainty about stroke diagnosis, combined with factors enabling rapid decision-making to appropriately test and treat, facilitate the presence of a significant subgroup of non-stroke patients in the care of stroke teams. This raises concerns for health policy about how to balance the benefits of a centralized services pathway with the realities that, in doing so, there will be patients with a variety of needs. Providers and planners should take better account of caring for these patients in terms of efficiency, cost-effectiveness, and quality of care. 
19.

\section{Quality of Emergency Room Diagnosis Determines Patient Outcome}

$\underline{\text { T. Birrenbach }}{ }^{1}$, M. Hoffmann 1 , S. C. Hautz ${ }^{1}$, J. E. Kämmer ${ }^{2}$, A. K. Exadaktylos ${ }^{1}$, T. C. Sauter ${ }^{1}$, W. E. Hautz ${ }^{1}$

${ }^{1}$ Inselspital, Bern University Hospital, Bern, Switzerland

${ }^{2}$ Charité Universitätsmedizin Berlin, Berlin, Germany

Background: Medical misdiagnosis is a major public health problem, causing increased morbidity and mortality. In the busy setting of an emergency department (ED), patients are diagnosed under difficult circumstances. The ED diagnosis at hospital admittance is often a descriptive or vague diagnosis, such as "decreased general condition" or may even be wrong. Previous studies have suggested patients presenting with non-specific complains (NSC) to be at an increased risk of misdiagnosis. Our objective was to determine whether patients with lowquality ED diagnosis experience a worse outcome than patients with a high-quality diagnosis.

Methods: We conducted a prospective observational study in one university-affiliated tertiary care hospital for all adult non-trauma patients admitted to any internal medicine ward. Patients' hospital discharge diagnosis was compared with the diagnosis at hospital admittance through the ED according to a predefined scheme. Low-quality diagnoses were defined as ED diagnoses experiencing a change in diagnosis or a vague ED diagnosis according to the "clinical classification software" for ICD-10, whereas high-quality diagnoses were defined as specific ED diagnoses, not undergoing a change in diagnosis. Furthermore we assessed length of hospital stay and in-hospital mortality.

Results: 755 consecutive patients were included, low-quality diagnoses were identified in 182 (24.1\%) of the cases. Low-quality diagnoses were significantly more likely to result in death $(\mathrm{OR}=2.13,95 \mathrm{CI}: 1.04-4.37 ; \mathrm{p}=0.034)$ and associated with lengthened stay in the ED (7.03h vs. $6.38 \mathrm{~h} ; \mathrm{p}=0.01$ ) but not in hospital. Patients presenting to the ED with nonspecific complaints (NSC) were more likely to receive a low-quality ED diagnosis ( $30.3 \%$ vs. $23.1 \%$; $p=0.039$ ), particularly if they were female ( $35.4 \%$ vs. $22.8 \%$; $=0.011$ ).

Conclusion: ED patients who receive a low quality diagnosis at hospital admittance have an increased risk of in-hospital mortality. Patients with NSC, especially women, are at an increased risk to receive a low-quality diagnosis. 
20.

\title{
Sensitivity of Chest X-Ray for Lung Cancer: Systematic Review
}

\author{
S. Bradley, A. Grice, B. Shinkins, $\underline{\text { R. D. Neal }}$
}

University of Leeds, Leeds, United Kingdom

Background: Lung cancer is a leading cause of premature death. Early diagnosis is associated with improved outcomes. While chest x-ray remains the first line investigation in many health care settings, individual studies have indicated that a proportion of lung cancers are not identified (false negatives); this is one cause of delayed diagnoses. Undetected cancers may be due to human error but in many cases lesions may be too small to be readily identified or they may be obscured by other anatomical structures on a two dimensional film. A normal chest $\mathrm{x}$-ray may falsely reassure both clinician and patient. The aims of this study was to estimate the proportion of patients with lung cancer who had a chest X-ray in the year prior to diagnosis which was not interpreted as having features suspicious for lung cancer, and therefore suffered a likely diagnostic error as a result.

Methods: In order to address the research question, a broad search strategy was devised including several synonyms for chest x-ray and lung cancer. Screening and paediatric data, studies from before 1999 and studies based on retrospective interpretation of radiology were excluded. A 20\% random sample was screened by a second reviewer to ensure consistency. The review has been registered with PROSPERO http://www. crd.york.ac.uk/PROSPERO/display_record.php?ID=CRD42017069629.

Results: Analysis is almost complete. Preliminary findings are that 8,362 studies were identified, and 29 studies met the inclusion criteria. Heterogeneity precluded meta-analysis, however the combined population was 1557 patients of whom 954 (61.27\%) had a positive chest x-ray. In three studies with a lower risk of bias, comprising 342 patients, sensitivity ranged from $73.5 \%$ to $79.3 \%$ (mean $76.2 \%$ ). These are shown in Table 1: Table 1

\begin{tabular}{lrrrr}
\hline Lead author & Year & n & True +ve & Sensitivity \% \\
\hline Bjerager & 2006 & 58 & 46 & 79.3 \\
Stapley & 2006 & 164 & 126 & 76.8 \\
Fernandez & 2007 & 102 & 75 & 73.5 \\
Combined & & 324 & 247 & 76.2 \\
\hline
\end{tabular}

Conclusion: This review suggests that chest x-ray does not initially identify approximately $25 \%$ of cases, although the findings may be affected by publication bias. This finding needs replicating in a high quality study that could also demonstrate the potential consequences on diagnostic intervals and subsequent stage at diagnosis. The findings also suggest that studies to evaluate the effectiveness and cost-effectiveness of other imaging modalities (e.g. low-dose CT scan) capable of identify smaller lesions (although they are will also identify nodules and other pathology of no clinical significance) are urgently needed. 
21.

\section{Teaching Reflective Reasoning through Explaining on Video}

$\mathrm{L} . \mathrm{Kuhn}^{1}$, S. Mamede', P. van den Berg'1, P. Bindels ${ }^{1}$, T. van Gog ${ }^{2}$

${ }^{1}$ Erasmus MC, Rotterdam, Netherlands

${ }^{2}$ University Utrecht, Utrecht, Netherlands

Background: Critical thinking is crucial in medical diagnostic. Automatic, non-analytical 'Type I' thinking (i.e., pattern recognition) is highly efficient with routine cases, but it also opens the door to error, for instance, when the initial diagnosis is accepted without much further thought. The method of structured reflection was developed as a means to enhance physicians' critical (Type II) thinking (Mamede, Schmidt, \& Penaforte, 2008, Medical Education). It has been shown that structured reflection can improve diagnostic accuracy on complex cases, can remedy diagnostic errors caused by cognitive bias, and can be a useful tool for training medical students' diagnostic competence. An open question, however, is whether the procedure can be also learned and applied when solving future, unrelated cases without instructions how to reason. A recent study found (Hoogerheide et al., in press), that students effectively acquired problem-solving skills in physics, when they first studied an example showing how to solve a problem, and then explained the learned content on video to a fictitious peer. We are currently preparing a study to investigate whether engaging in video explaining is also effective for learning the procedure of structured reflection.

Methods: Ninety residents in general practice will be randomly assigned to one of two conditions in the learning phase. In the control condition, participants diagnose six cases without further instructions. Participants in the explanation condition first study three examples of cases solved with structured reflection. After this, they make two web-cam recordings in which they explain to a fictitious peer (1) the steps of structured reflection, and (2) how to apply it on a given case. On a delayed test (one week later), all participants will diagnose a new set of 12 cases. Time on task will be measured on each test case.

Results: It is hypothesised that participants in the explanation condition will learn the procedure and thus engage in more reflective reasoning compared to the control condition, leading to more time on task and higher diagnostic accuracy.

Conclusion: The results of this study will contribute to the development of guidelines for fostering critical thinking in general medical education, which may be applicable in other professional domains as well. 
22.

\section{The Morbidity and Mortality Conference As a Tool to Reveal Diagnostic Errors}

B. Frey, V. Bernet, B. Brotschi, V. Cannizzaro, C. Doell

University Children's Hospital, Zürich, Switzerland

Background: The morbidity and mortality conference (MMC) served traditionally as an educational aid for medical trainees, but more recently, new emphasis has been placed on patient safety. It has been shown that the MMC is superior to chart review in identifying problems, such as miscommunication, workflow issues, and certain diagnostic errors [1]. We analysed our MMCs regarding the identification of diagnostic errors.

Methods: This is a retrospective analysis of the minutes of the MMCs held from January 2009 to June 2014, in a 23-bed tertiary interdisciplinary pediatric intensive care unit [2]. Qualifying cases are deaths and major incidents, regardless of possible medical errors. Moderators have experience in error and risk analysis. We analyzed the protocols with respect to: morbidity/mortality, patient characteristics, and autopsy data as a pointer to relevant new diagnoses classified by Goldman criteria. Wherever the possibility of error was raised, we analyzed whether it met with participant consensus. Error categories included diagnosis, ventilator, invasive device, medical or surgical procedure, infection, drugs, and miscommunication. We attributed to each error the most appropriate root cause (individual or system-based).

Results: 48 MMCs were held over the 5.5 years, $73 \%$ of whom underwent autopsy. Principal diagnoses: cardiac 18, respiratory 6, perinatal asphyxia 6, congenital malformation (other than heart) 5, septic shock 3, cerebral disease 3, solid neoplasm 1, inborn error of metabolism 1, trauma 1, and hemorrhage (other than brain, not traumatic) 1. In 77\% of MMCS 50 unsafe processes/medical errors were discussed and identified, including 12 diagnostic errors. The diagnostic errors are detailed in the Table. In 3 cases the correct diagnosis was established too late, in one case a detailed neurologic evaluation in a disabled child was not performed preoperatively and in the remaining 8 instances, the deterioration of vital signs was diagnosed too late. In two patients, the autopsy revealed relevant new diagnoses.

Conclusion: The interdisciplinary and interprofessional morbidity and mortality conference has the potential to reveal unsafe processes/ medical errors, especially diagnostic errors. In our setting of pediatric intensive care, most diagnostic errors were related to late diagnosis of deterioration. References: 1. Ciffra CL, Jones KL, Ascenzi J, et al. The morbidity and mortality conference as an adverse event surveillance tool in a paediatric intensive care unit. BMJ Qual Saf 2014, 23:930-938. 2. Frey B, Doell C, Klauwer D, et al. The morbidity and mortality conference in pediatric intensive care as a means for improving patient safety. Pediatr Crit Care Med 2016, 17:67-72.

\begin{tabular}{|c|c|c|c|c|c|c|c|}
\hline $\begin{array}{l}\text { Morbidity/ } \\
\text { Mortality }\end{array}$ & Age & Principal diagnosis & Diagnostic error & $\begin{array}{l}\text { Root cause } \\
\text { (individual or } \\
\text { system-based } \\
\text { error) }\end{array}$ & $\begin{array}{l}\text { Interface } \\
\text { problem }\end{array}$ & $\begin{array}{l}\text { Autopsy withrelevant } \\
\text { new diagnoses }\end{array}$ & $\begin{array}{l}\text { Corrective } \\
\text { interventions }\end{array}$ \\
\hline Mortality & $7 y$ & $\begin{array}{l}\text { Urea cycle defects (inborn error of } \\
\text { metabolism) }\end{array}$ & Diagnosis too late & Individual & yes & Not performed & In hospital \\
\hline Mortality & $17 y$ & Cerebral hemorrhage, spontaneous & $\begin{array}{l}\text { Diagnosis too late, warning signs } \\
\text { overseen }\end{array}$ & System-based & yes & Not performed & In hospital \\
\hline Mortality & $4 \mathrm{mo}$ & $\begin{array}{l}\text { Unbalanced atrioventricular septal } \\
\text { defect, failing Fontan }\end{array}$ & $\begin{array}{l}\text { Hypoxemia not acknowledged } \\
\text { andcardiac catheter too late }\end{array}$ & System-based & yes & Not performed & In hospital \\
\hline Morbidity & $7 \mathrm{~d}$ & Congenital solid tumor in epipharynx & Correct biopsy result too late & System-based & yes & No new diagnoses & $\begin{array}{l}\text { In PICU, hospital } \\
\text { and outside }\end{array}$ \\
\hline \multirow[t]{3}{*}{ Mortality } & \multirow[t]{3}{*}{$6 y$} & \multirow[t]{3}{*}{ Multiple disabilities, cardiac arrhythmia } & No preoperative neurologic evaluation & System-based & & \multirow[t]{3}{*}{ Not performed } & \\
\hline & & & Early discharge from PICU & individual & & & In hospital \\
\hline & & & Deterioration not diagnosed & System-based & & & In hospital \\
\hline Mortality & $2.5 y$ & Trisomy 21, pulmonary hypertension & $\begin{array}{l}\text { Severity of pulmonary hypertension } \\
\text { was not diagnosed before intervention }\end{array}$ & System-based & yes & Not performed & In hospital \\
\hline Mortality & $4 \mathrm{mo}$ & $\begin{array}{l}\text { Atrioventricular septal defect, pulmonary } \\
\text { hypertension }\end{array}$ & $\begin{array}{l}\text { Severity of pulmonary hypertension } \\
\text { was not diagnosed before intervention }\end{array}$ & System-based & yes & $\begin{array}{l}\text { Signs of severe } \\
\text { pulmonary } \\
\text { hypertension, lung } \\
\text { embolism }\end{array}$ & In hospital \\
\hline Mortality & $8 \mathrm{mo}$ & Ebstein anomaly & $\begin{array}{l}\text { Severity of disease was not diagnosed } \\
\text { in other hospital (transfer too late) }\end{array}$ & System-based & yes & CMV infection & $\begin{array}{l}\text { In hospital and } \\
\text { outside }\end{array}$ \\
\hline Mortality & $4 y$ & $\begin{array}{l}\text { Septic granulomatosis, nocardiosis, } \\
\text { ARDS }\end{array}$ & $\begin{array}{l}\text { Severity of disease was not diagnosed } \\
\text { in other hospital (transfer too late) }\end{array}$ & Individual & yes & No new diagnoses & In PICU \\
\hline Mortality & $16 y$ & Disseminated tuberculosis & Diagnosis too late & Individual & & Not performed & In hospital \\
\hline
\end{tabular}


23.

\title{
The Patient, the Case, the Physician and Their Context: A Structural Equation Model to Assess the Quality of Team Performance and the Effect on Patient Outcome in Emergency Care
}

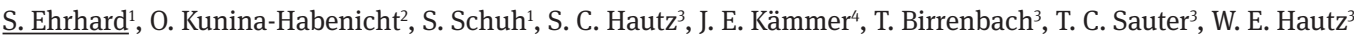 \\ ${ }^{1}$ Inselspital University Hospital Bern, Bern, Switzerland \\ ${ }^{2}$ University of Education Karlsruhe, Karlsruhe, Germany \\ ${ }^{3}$ Inselspital, Bern University Hospital, Bern, Switzerland \\ ${ }^{4}$ Charité Universitätsmedizin Berlin, Berlin, Germany
}

Background: Evidence suggests that improving teamwork is key to reduce the rate of error in emergency medicine but little is known about factors affecting the performance of diagnostic ad hoc teams. The majority of previous studies have been conducted in controlled experimental settings and with often rather homogeneous teams. The aim of this study was thus to determine mechanisms that result in high-quality teamwork of ad hoc teams and its impact on patient outcome.

Methods: Prospective, observational study at a university emergency department. Variables describing the patients, diagnosing physicians and the context were collected. Each patient was seen by a resident and an attending physician. Both completed a questionnaire each and per patient. A structural equation model (SEM) was used to simultaneously model latent relationships between different doctors' characteristics on the individual and team level, patients' characteristics, context and patient outcome. We postulated separate latent factors for case difficulty, quality of teamwork, and work experience for each doctor. Objective workload was captured in a separate latent factor consisting of department crowding and noise level. Finally, a factor for urgency was modeled using triage category and treatment in a resuscitation bay. Results: The final SEM revealed a good model fit $\left(\mathrm{X}^{2}=322.66 ; \mathrm{df}=244 ; \mathrm{p}<.001 ; \mathrm{CFI}=.935\right.$; RMSEA $\left.=.026\right)$. While case difficulty ( $\left.\mathrm{r}=.63\right)$ and quality of teamwork $(\mathrm{r}=.73)$ were evaluated similarly by attending physician and resident, we found substantial differences in their evaluations of factors outside the team. For example, the urgency of treatment of a patient was positively related to case difficulty rated by the attending $(\mathrm{r}=.28)$, as was quality of teamwork $(\mathrm{r}=.28)$, while none of the latent residents factors correlated with urgency. For residents, objective workload correlated negatively with case difficulty $(\mathrm{r}=-.21)$ and quality of teamwork $(\mathrm{r}=-.19)$. We did not find any significant correlation between teamwork and patient outcome.

Conclusion: Although high-quality teamwork is suspected to be an important factor to reduce diagnostic error, this study did not find any relationship between several descriptors of teamwork and patient outcome. However, we found interesting differences regarding the factors used for the evaluation of teamwork quality and case difficulty between the members of the diagnostic team: while urgency of treatment of the patient affects experienced physicians' evaluation of case and teamwork, junior doctors' evaluations seem to be more susceptible to changes in workload. Future studies of diagnostic teamwork should thus pay attention to the individual differences between members of diagnostic teams. 
24.

\title{
Understanding Diagnostic Reasoning Using a Case-Based Approach
}

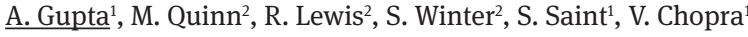 \\ ${ }^{1}$ VA Ann Arbor Healthcare System, Ann Arbor, MI \\ ${ }^{2}$ University of Michigan, Ann Arbor, MI
}

Background: While understanding the cognitive contributions to diagnosis has become increasingly important, few studies have aimed to understand physician diagnostic reasoning in real-time. Using a case-based approach, we sought to: (a) characterize differences in diagnostic reasoning among hospitalists; and (b) identify factors associated with diagnostic accuracy.

Methods: A total of 10 hospital medicine attending physicians from an academic medical center took part in the case-based interview. Hospitalists were provided pertinent information regarding a patient's presentation in a step-wise fashion (Figure), and asked to formulate a differential diagnosis following each new piece of key data. Following presentation of basic labs, participants were able to request results for additional studies, which were provided if performed. An updated clinical course was also provided to participants regularly. All sessions were audio-recorded and transcribed verbatim. Synthesis of quantitative and qualitative data using mixed methods were used to identify key findings by two reviewers (AG, VC).

Results: Ten hospitalists ( 5 male and 5 female) participated in interviews. The average years post-residency was 7.2 (range 2-16 years. The correct diagnosis (shingles) was included in the final differential of 4 of the 10 participants. Hospitalists that identified the correct answer differed from those that did not in several ways. First, hospitalists that included the correct diagnosis included more diagnoses in their initial differentials (mean 9.75 [range 8-13, SD 1.18] vs 6.0 [range 4.0-10.0, SD 0.89], $\mathrm{p}=0.03$ ). Second, those that included the correct diagnosis also used a specific organizational structure to generate candidate diagnoses, including systems-based (i.e., cardiovascular, pulmonary) and anatomical (i.e., chest, abdomen) approaches. Third, while all hospitalists prioritized life-threatening diagnoses, those whose initial differential included only those causes were less likely to arrive at the correct diagnosis and more likely to demonstrate anchoring further into the case. Finally, those correctly identifying the diagnosis frequently re-examined data regarding the patient's initial presentation or physical examination.

Conclusion: Although limited to a small sample size from one academic medical center, particular behaviors in diagnostic evaluation appear to limit anchoring and improve diagnostic accuracy. Frequent and intentional re-evaluation of data in the context of the patient's initial presentation are likely important in refining differential diagnoses. 
Figure: Flow diagram of case-scenario progression (received by all participants)

Chief Complaint: 66 yoM with history of tobacco abuse presents with chest pain

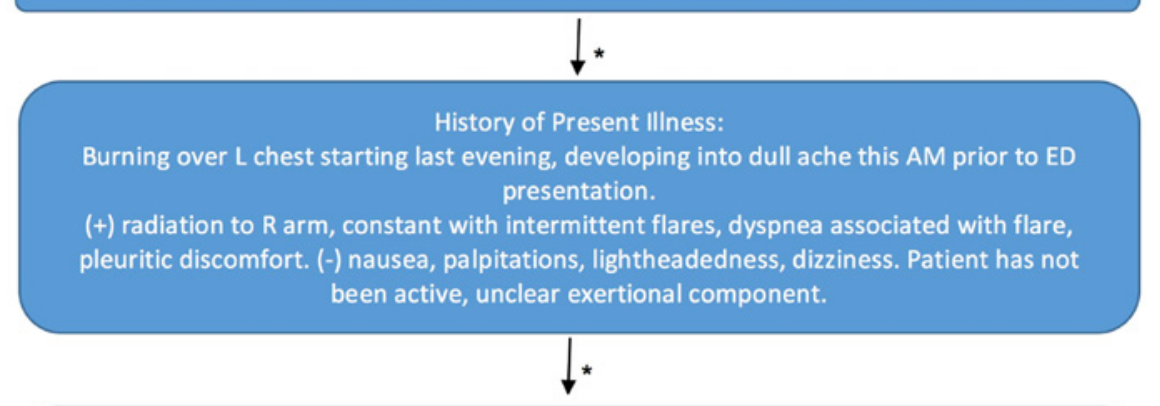

Past Medical/Surgical History: Hypertension, tobacco abuse

Medications: Metoprolol $25 \mathrm{mg}$ twice daily

Family History: Father with MI at age 55, mother with diabetes, hypertension

Social History: Lives with wife and 2 daughters; smokes 1 pack/day $\times 35$ years, Denies

alcohol or illicit drug use; retired autoworker

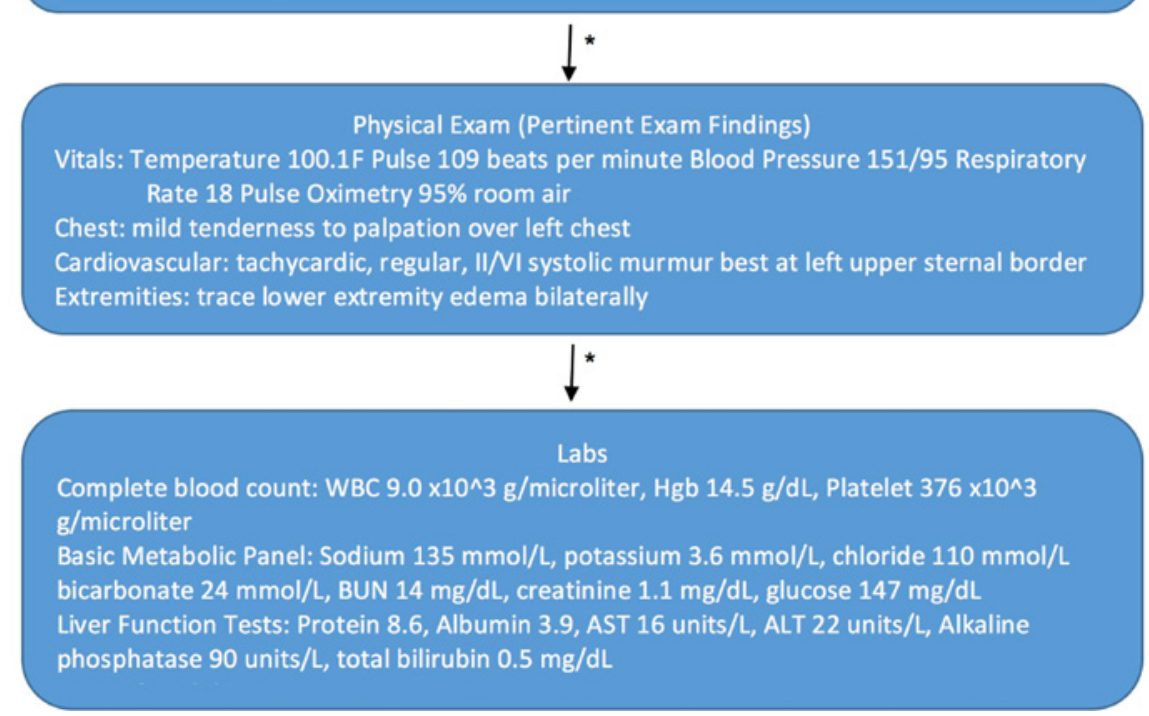


25.

\title{
Understanding Systems-Based and Cognitive Contributions to Diagnosis
}

\author{
$\underline{\text { A. Gupta }}^{1}$, M. Harrod ${ }^{1}$, M. Quinn ${ }^{2}$, K. Fowler ${ }^{1}$, S. Saint ${ }^{1}$, V. Chopra ${ }^{1}$ \\ ${ }^{1}$ VA Ann Arbor Healthcare System, Ann Arbor, MI \\ ${ }^{2}$ University of Michigan, Ann Arbor, MI
}

Background: Diagnostic error in medicine is increasingly recognized as "the next frontier for patient safety". Current research has focused on diagnostic errors in two unique dimensions: as systems-based or cognitive-based etiologies. One study, however, suggests that nearly half of all errors stem from both domains. Using a focused ethnographic approach, we sought to understand how systems-based factors contribute to the cognitive missteps underlying diagnostic error.

Methods: We conducted a focused ethnography of inpatient medicine teaching teams at two affiliated academic medical centers. Teams (consisting of 1 attending, 1 senior resident, 2-3 interns, and 1-4 medical students) were observed on rounds and during post-round work. Field notes related to the diagnostic process as well as the work system were recorded and collated. Observations were followed by focus groups with interns and resients and interviews with attending physicians, to better understand perceptions around diagnosis and factors contributing to error. Inductive analysis was used to organize findings into themes, which were then categorized according to a previously described taxonomy into systems-factors contributing to the cognitive domains of faulty data gathering, faulty information processing, and faulty data verification. An additional category describing work environment factors encompassing all of these cognitive domains was included.

Results: Between January 2016 and April 2017, 10 teams were observed over the course of 286 hours. All 10 attendings and 31 interns and residents participated in interviews or focus groups. The following key themes that bridged system and cognitive factors were identified. First, challenges with interdisciplinary communication, particularly in-person communication, as well as closed-loop communication within the electronic medical record (EMR) were felt to contribute to faulty data gathering. Second, information contained within the EMR was often considered unreliable and inaccessible due to poor integration and interface. Third, organizational structures such as the operation of consulting services in silos and the reliance on inexperienced overnight radiology interpretations were felt to contribute to faulty information processing. Fourth, frequent care handoffs, between inpatient providers as well as the emergency department and the inpatient ward, were noted to provide opportunities for faulty data verification. Finally, interruptions, time constraints, and a cluttered physical environment were found to adversely impact all cognitive domains.

Conclusion: Systems-based and cognitive factors likely occur simultaneously in diagnostic errors. Interventions aimed at addressing cognitive contributions should be coupled with systems-based interventions in order to maximize effectiveness. 
26.

\section{Unravelling Information Processing in Correct and Incorrect Diagnosis Using Eye-Tracking - a Study Design}

L. Staal, J. N. Van der Geest, J. Alsma, M. A. Frens, L. Zwaan

Erasmus MC, Rotterdam, Netherlands

Background: Diagnostic errors are believed to be caused by cognitive biases and thought to arise due to fast diagnostic reasoning and selective information processing. Several interventions have been developed to 'debias' physicians and recommendations involve slowing down the diagnostic process and reconsidering a diagnosis. Conversely, there is an increasing amount of evidence that it is content knowledge rather than the reasoning process that is the main cause of diagnostic errors. We describe the design of a study that challenges the idea that diagnostic errors are the result of fast and selective reasoning and will test the hypothesis that fast and selective reasoning also occurs in correctly diagnosed cases.

Methods: Thirty medical residents will solve 6 clinical cases to induce availability bias (phase 1) in a similar way as shown in an earlier study by Mamede et al. Subsequently, participants will diagnose 8 cases (phase 2) of which four cases are similar to the biased cases. An expert panel will identify the features that are relevant and irrelevant to the correct diagnosis for all clinical cases. Using eye-tracking we will identify how often participants looked at those features, how often they returned to those features, and how much time they spent looking at those features. All measures will be compared between correctly and incorrectly diagnosed cases (both due to availability bias and other diagnostic errors).

Results: The study has not yet been carried out, so there are no results available yet.

Conclusion: We expect that selectivity in the reasoning process will take place in cases with and without diagnostic errors and cases with cognitive bias. We expect that the difference between correctly and incorrectly diagnosed and biased cases will not be due to considering less information, but due to a focus on irrelevant information. Additionally, we expect that correctly diagnosed cases are diagnosed faster than cases with diagnostic errors or cognitive biases. This study will contribute to the understanding of how diagnostic errors occur. 


\title{
27.
}

\section{Voluntary Use of Guided Reflection for Diagnostic Reasoning in Psychiatry: A Think-Aloud Protocol with Junior Doctors}

\author{
$\underline{\text { K. Lambe }}{ }^{1}$, D. Hevey², B. D. Kelly ${ }^{3}$ \\ ${ }^{1}$ Trinity College Dublin, Dublin, Ireland \\ ${ }^{2}$ School of Psychology, Dublin, Ireland \\ ${ }^{3}$ Trinity Centre for Health Sciences, Dublin, Ireland
}

Background: Reflective interventions have been shown to be beneficial for diagnostic reasoning. Empirical studies of guided reflection interventions, which encourage doctors to take a step-by-step analytical approach and to gather supporting and refuting evidence for one or more diagnostic hypotheses, have largely comprised quantitative experiments, with relatively little consideration of how doctors may voluntarily use the guided reflection process and the specific ways in which it changes or enhances their diagnostic process. Additionally, the study of diagnostic reasoning and error within psychiatry has been largely neglected in the broader literature. This study therefore utilised a think-aloud protocol to observe how junior doctors made voluntary use of a guided reflection tool in diagnosing three complex psychiatric clinical scenarios.

Methods: Participants were asked to diagnose three fictional clinical cases aloud and were provided with the guided reflection process as an additional tool, which they could use or not use at their discretion. A semi-structured interview was utilised to supplement the think-aloud procedure. Participants were 14 junior doctors, all of whom were undertaking Basic Specialist Training $(n=10)$ or Higher Specialist Training $(n=1)$ in psychiatry or Specialist Training in general practice $(n=3)$.

Results: A majority of participants chose to use the tool and engaged with all of the steps outlined in the process. Participants responded positively to the process, describing it as simple and easy to use. Thematic analysis revealed that guided reflection helps doctors to slow down and expand their diagnostic thinking. Participants said that the process closely reflects their own natural diagnostic process, but that it provides visible structure to diagnostic activities they would normally undertake. Participants reported that use of the process increased their confidence; however, participants rarely changed their minds from their very first diagnostic hypothesis. Participants broadly indicated that the tool had potential for use in real-world diagnosis, particularly for ambiguous or complex cases.

Conclusions: Given the option under laboratory conditions, doctors made use of guided reflection in most cases, with case complexity being a key driver of this choice, and they regard the intervention as straightforward and easy to use. Think-aloud protocols can offer rich insights into not only the outcomes but also the processes of cognitive interventions. Continued research with more sophisticated techniques will further develop our understanding of what works to improve diagnostic reasoning - and why. 
28.

\title{
Which Diagnoses are Missed in Primary Care and in Emergency Departments?
}

\author{
$\underline{\text { R. Fernholm }}$ \\ Karolinska Institutet, Huddinge, Sweden
}

Background: Patient safety research has for quite some time had its focus on the hospital setting, for example complications within surgery and hospital acquired infections. In recent years, the importance of patient safety in the primary care setting has been highlighted by the work of WHO and others. Patient harm in primary care can for example be a result from missed diagnoses of cancer or myocardial infarction, where the patient does not receive treatment in time. Primary care is usually the first contact with the health care system and has a big responsibility to offer safe and high quality care. Primary care works with a high degree of diagnostic uncertainty and so does the emergency departments. Our aim is to explore what diagnoses are missed in these settings.

Methods: Cross sectional study of a Swedish registry called LÖF. The registry consists of avoidable patient harm (patients report to the registry for financial compensation) and from the years 2011-2016 there were 2897 cases from primary care and 1249 cases from emergency departments. The cases were analysed to explore what diagnoses were missed.

Results: A missed diagnosis was registered as the cause in $42 \%$ of all cases in primary care and in $41 \%$ of all cases in the emergency departments. The most commonly missed diagnoses in primary care were: cancer 25\% (mostly colorectal), fractures 14\% (mostly hand/wrist), muscle/tendon injuries 12\% (mostly Achilles and rotator cuff) and infections 11\% (mostly soft tissue). The most commonly missed diagnoses in emergency departments were: fractures 28\% (mostly hand/wrist), muscle/tendon injuries 22\% (mostly fingers) and infections 11\% (mostly appendicitis).

Conclusion: Diagnostic errors dominate patient safety events in primary care and in emergency departments. Cancer is the most commonly missed serious diagnoses in primary care while fractures dominate in the emergency department. With the knowledge of what diagnoses that are commonly missed, we believe that the task to prevent missed diagnoses will be facilitated, and that it will be feasible to work for a reduction of missed diagnoses and thereby decrease the risk of patient harm. 
29.

Young Doctors Facing Medical Errors, a Possible Solution to Control the Incidence of Missed Diagnostics

D. V. Turcu ${ }^{1,2}$, S. C. Dupa ${ }^{2,3}$, P. Salahoru ${ }^{4,5}$, T. Mihaescu $u^{4,5}$

${ }^{1}$ Clinic of Respiratory Diseases, Iasi, Romania

2"Grigore T. Popa” University of Medicine and Pharmacy, Iași,Romania, Iasi, Romania

3“Sfanta Maria” Emergency Hospital for Children, Iasi, Romania, Iasi, Romania

${ }^{4}$ Clinic of Pulmonary Diseases, Iasi, Romania

5“Grigore T. Popa” University of Medicine and Pharmacy, Iasi, Romania

Background: Patient safety is paramount for a reliable and qualitative medical system. In spite of the fact that the topic of medical errors has been in debate for many years, they are still responsible for a great number of fatalities around the world. The first place in this hierarchy is taken by the diagnostic errors. Cognition is the main contributor for this type of errors. The purpose of the study is to assess how medical residents are instructed to use cognition in the process of clinical reasoning.

Methods: The study consisted in a 14-items online questionnaire which was applied to 51 male and female medical residents in Iasi, Romania, between the dates of July $4^{\text {th }}$ and September $4^{\text {th }} 2017$.

Results: A big majority of respondents (84.2\%) have agreed to the existence of cognitive biases throughout the diagnostic process. Nearly half of the contributing residents (41.2\%) believe that the experience of older doctors is aiding them to commit less cognitive errors. The same margin of respondents recognizes that they do not have the necessary knowledge to reduce the incidence of diagnostic errors.

Conclusion: The survey showcases a very real hiatus of information required to prevent diagnostic errors. Another point of reference is the necessity for educating the clinical reasoning of resident doctors. 


\section{Applied Innovations}

\section{Poster Session 1 - Applied Innovations}

30.

\section{Development of Standardised Recommendations Concerning Blood Drawing for Coagulation Testing for Health Professionals in Tyrol}

H. Oberhauser, M. Bachlechner

Health University of Applied Sciences Tyrol, Innsbruck, Austria

Statement of problem: Different studies show, that errors in laboratory diagnostics are in the most cases (46\%-68,2\%) due to mistakes in the preanalytical phase. Errors in laboratory testing lead to increasing healthcare expenses and could have direct consequences to the patient (Cornes et al. 2017). In fact that blood drawing is usually carried out outside of the control of the laboratory and from different health professionals (e.g. nurses, pathologists, biomedical scientists, medical assistant professions), the knowledge about correct blood sampling is of utmost importance. Partly contradictionary statements concerning blood drawing for coagulation testing, especially regarding filling level, order of drawing and usage of discard tubes, complicate the blood drawing process (Meyer et al. 2017). There is evidence, that knowledge and awareness of blood drawing recommendations are lacking among health care professionals outside the laboratory (Söderberg et al. 2009). Therefore the aim of this study is to standardise the blood drawing procedure for coagulation testing in Tyrol.

Description of the intervention or program: Via online-questionnaire teachers and students of different educational institutes for health professionals in Tyrol are asked about instructed regulations concerning blood drawing for coagulation testing. Hospital wards are asked about blood drawing sets and recommendations used and groups of health professionals who are carrying out the blood drawing. Laboratories are asked about criteria and percentage of refusal of samples because of inadequate filling level. A practicable quick reference guide for education of health professionals and professional practitioners in Tyrol will be developed.

Findings to date: It is to assume, that recommendations of blood drawing differ in educational institutes and hospitals. Through actively involving of teachers, students and professional practitioners, a high willingness for blood drawing due to current state (Meyer et al. 2017) is expected.

Lessons learned: Correct blood drawing is an essential condition for proper laboratory results. Therefore it is important, that all involved groups of health professionals are informed about the process and possible consequences. Actively involving of apprentices and practitioners increase the implementation of evidence-based decisions into clinical Routine. 
31.

\title{
Emotions of Medical Students When Learning with Virtual Patients
}

\author{
J. Kiesewetter ${ }^{1}$, R. Schönberger ${ }^{1}$, V. Jung ${ }^{1}$, M. Sailer², L. Zottmann ${ }^{3}$, F. Fischer², M. R. Fischer ${ }^{1}$ \\ ${ }^{1}$ Klinikum der LMU München, Munich, Germany \\ ${ }^{2}$ LMU München, Munich, Germany \\ ${ }^{3}$ University hospital of LMU Munich, Munich, Germany
}

Statement of problem: One key goal of medical schools is to foster clinical reasoning skills. Virtual patients (VPs) play an increasingly important role in medical education in general and in the training of clinical reasoning in particular. It has been shown that virtual patients can help students to learn clinical accuracy. This study investigates, which emotions are associated with learning of clinical reasoning with VPs. Our research question is: How do emotions relate to diagnostic performance indicators?

Description of the intervention or program: In a computer lab, medical students of the $3^{\text {rd }}$ to the $6^{\text {th }}$ clinical year had to diagnose eight VPs in the learning environment CASUS. Overall, 142 medical students participated in this study ( $72 \%$ female, age $M=24.41$ years). As performance indicators diagnostic accuracy and time-on-task were implemented. Diagnostic accuracy was defined as the sum of correct diagnoses with respect to the eight VPs (1 point per correct diagnosis, no partial credit). Time-on-task was measured as seconds from the beginning of reading the first VP information to the entering of the diagnosis. After four and after eight VPs students rated their emotions on a 5-point Likert scale. We asked for positive (curiosity, surprise, enthusiasm) and negative (confusion, anxiety, frustration) emotions and boredom. Alpha-error level was set to $\mathrm{p}<.05$, two-sided for Pearson correlations.

Findings to date: The correlations show a negative linear relationship between diagnostic accuracy and the negative emotions anxiety $(\mathrm{r}=-.33, \mathrm{p}=.00)$, frustration $(\mathrm{r}=-.35, \mathrm{p}=.00)$ and confusion $(\mathrm{r}=-.26, \mathrm{p}=.00)$ after four VPs and anxiety $(\mathrm{r}=-.39, \mathrm{p}=.00)$, frustration $(\mathrm{r}=-.32$, $\mathrm{p}=.00)$ and confusion $(\mathrm{r}=-.20, \mathrm{p}=.01)$ after eight VPs. Additionally, the correlation between frustration and time-on-task was significant after four VPs $(\mathrm{r}=.18, \mathrm{p}=.03)$ and after eight VPs $(\mathrm{r}=.19, \mathrm{p} .=.02)$. The correlations between the positive emotions and diagnostic accuracy as well as between the positive emotions and time-on-task were not significant after four and eight VPs. Boredom had no significant correlations in any analysis.

Lessons learned: This study has shown that students affective experiences of negative emotions during VP encounters negatively relates to their diagnostic accuracy in reasoning processes. As correlations allow no casual interpretation, the question whether negative emotions imply low diagnostic accuracy or low diagnostic accuracy results in negative emotions has to be investigated further. Additionally, confusion is related to higher time-on-task. These findings raise the question whether all students are equally able to profit from learning with VPs. These findings suggest to carefully design VPs, which are not emotionally charged, in particular regarding negative emotions. 
32.

\section{Exploiting Predictive Brains for Better Diagnosis}

\section{T. K. LIM, C. M. Teoh}

National University Hospital, Singapore, Singapore

Statement of problem: Studies which applied the dual process model of cognition to improve diagnosis by "de-biasing” have yielded disappointing results. Thus, it might be appropriate to consider alternative models of cognition based on advances in neuroscience such as the predictive brain. The predictive brain is characterised as a rapidly adaptive, generative, energy-frugal, context-sensitive action-orientated, probabilistic, cognitive engine dedicated to processing predictive errors (PE). This system is regulated and fine-tuned by varying the gains on the weightage of the reliability(or precision) of the PE themselves. This capacity to effectively manipulate and re-calibrate the precision of PE (i.e. level of confidence Vs uncertainty) may be a critical aspect of clinical problem solving and learning.

Description of the intervention or program: Internal medicine residents were instructed to note and append the following features to their clerking impression in the patient's electronic health record (eHR): (a) Counter diagnostic features; (b) Things that do not fit; (c) Red flags. "Counter diagnostic features" were symptoms, signs or investigations which were inconsistent with the initial diagnosis. "Things that do not fit" were any findings that could not be accounted for. "Red flags" were findings which raised the possibility of a more serious underlying illness requiring early diagnosis or intervention. The attending physicians gave feedback on these points during bedside rounds and made amendments as appropriate.

Findings to date: We describe the first 128 patients (77 intervention Vs 51 controls) who had completed templates documented in their eHR. Residents documented "Counter diagnostic features" in 73\% Vs 51\% ( $p=0.015)$, “Things that do not fit" in 78\% Vs 24\% ( $p=0.002)$, and "Red flags" in $83 \%$ Vs $24 \%$ ( $p=0.0001$ ) of cases respectively. Frequently cited "counter diagnostic features" and "red flags" were pneumonia without chest radiographic evidence of airspace consolidation and haemoptysis respectively. Qualitative feedback suggests that this template enhanced supervisor-trainee interaction, improved diagnostic accuracy, and prevented diagnostic pitfalls without significant increasing the time required to complete the ward rounds. Some residents continued to enter pitfall points in the eHR long after the initial 4 week posting when this was introduced.

Lessons learned: This exercise gives us an opportunity to see if we can improve diagnostic accuracy by using pivotal words-to-self in the appropriate setting to maintain cognitive openness, flexibility and thus, avoid premature closure. It is also a valuable critical thinking habit to inculcate in tyro diagnosticians. Exploiting predictive brains by employing language to manipulate the precision-weighting of our own PE may be a way forward to reducing diagnostic errors. 
33.

\title{
How Do Patients and Carers Want to be Involved in Primary Care Patient Safety? a Qualitative Study Examining the Role of a Patient Safety Guide in Primary Care
}

\author{
$\underline{\text { R. L. Morris }}{ }^{1}$, S. Giles ${ }^{2}$, S. Campbell ${ }^{2}$ \\ ${ }^{1}$ University of Manchester, UK, Manchester, United Kingdom \\ ${ }^{2}$ University of Manchester, Manchester, United Kingdom
}

Statement of problem: Increasingly support is given to person-centred approaches in health and social care which empower patients and carers to be involved actively in their patient safety. A key part of this is considering the role of patients in missed diagnostic opportunities. This involves not only asking patients to identify safety concerns and errors, but also to partner in preventing them. Co-design approaches have been used to develop, implement and evaluate participatory approaches in quality improvement initiatives by working in collaboration with the end-users of the intended initiatives. This project used a co-production approach to develop a primary care patient safety guide for patients and carers to support their active involvement in patient safety.

Description of the intervention or program: A co-design approach was used to develop a prototype of the patient safety guide (including paper and online versions). After an initial brief scoping exercise of patient safety issues was conducted, 2 co-design events with 30 participants per event were took place with a mix of General Practitioners, pharmacists, patients and carers. During the events participants mapped the phases of care in relation to patient safety in priority areas, planned ways of improving patient safety at key points of care, and then worked together to refine the guide. Following the co-design events, semi-structured interviews with 19 patients and carers were conducted to examine patients and carers existing strategies for patient safety and to evaluate the guide.

Findings to date: Healthcare professionals, patients, carers, and researchers working together to co-design the patient safety guide enabled the identification of shared priority areas and patient safety questions to address, such as developing a shared understanding of patient safety, understanding each other's role in safety and diagnosis, and to support collaborative improvement. The qualitative study identified a range of patient or carer factors that could influence their engagement in patient safety; such as frequency of contact with services, acute compared to long-term condition management, and communication with healthcare professionals.

Lessons learned: Using a co-production approach and in-depth qualitative study identified important areas where patient safety in routine primary care. This study provides a more nuanced understanding of how patients and carers want to be involved in patient safety reflected in a continuum of expectations of care and everyday priorities. The guide has the potential to support patients and carers to optimise their use of services and be active in their patient safety. 
34.

\title{
Identification and Management of Medical Error in Primary Care: A Simplified Tool for Root Cause Analysis
}

\author{
A. Gouveia, P. Staeger \\ University of Lausanne, Lausanne, Switzerland
}

Statement of problem: Medical errors threaten patient safety, including when patients are seen in the primary care physician's office. The ambulatory setting presents several particularities with regard to errors and their management. Practitioners' solitude has an important weight on the capacity to face an error, imposes a transparent and systemic management of the situation and needs the active demand for peer support.

Description of the intervention or program: Root cause analysis is a valid approach for assessing error in the medical field, however it must be adapted to the context of general practice and family medicine. In this presentation, we propose a simplified tool for conducting root cause analysis in primary care by family physicians. Three fundamental questions for the identification and management of the error are key in this process: how did it happen, what happened and why did it happen. The proposed grid will make it possible to identify the error in a clear way, to unfold the facts behind the error itself, and to recognize the system failures (latent conditions, error-producing conditions and faults in the defenses).

Findings to date: After accomplishing the development of the tool, we have started the implementation phase by our GP trainees and chief residents working in our department, as a way to present and to interpret diagnostic error in the morbidity and mortality conferences that we conduct at our center. We estimate that the improvement measures that will result from each case, as well as the acquisition of expertise in error identification and management, will have a positive impact in our team regarding the improvement of diagnostic skills and the prevention of diagnostic errors.

Lessons learned: Adding to the application of the proposed tool for root cause analysis, we consider to be of utmost importance the dissemination of root cause analysis results in order to prevent further medical errors. With our simplified tool, general practitioners will easily acquire skills and feel better supported in the identification and management of errors in the primary care context, while learning from each other. 


\begin{tabular}{|c|c|c|c|c|}
\hline $\begin{array}{l}\text { How did it } \\
\text { happen? } \\
\text { (timeline of events) }\end{array}$ & $\begin{array}{l}\text { A healthy } 40 \text {-year } \\
\text { old patient calls a } \\
\text { friend doctor } \\
\text { because of an } \\
\text { acute episode of } \\
\text { left facial pain } \\
\text { with numbness } \\
\text { and a watering } \\
\text { eye. The family } \\
\text { doctor adresses } \\
\text { the patient to the } \\
\text { hospital } \\
\text { suspecting of } \\
\text { trigeminal } \\
\text { neuralgia. }\end{array}$ & $\begin{array}{l}\text { At the } \\
\text { emergency } \\
\text { room, the } \\
\text { diagnosis of } \\
\text { trigeminal } \\
\text { neuralgia is } \\
\text { retained after a } \\
\text { specialized } \\
\text { assessment done } \\
\text { by a consultant } \\
\text { neurologist. } \\
\text { Carbamazepine } \\
\text { was prescribed. }\end{array}$ & $\begin{array}{l}\text { After the } \\
\text { treatment was } \\
\text { started, the patient } \\
\text { developed } \\
\text { sleepiness and } \\
\text { became febrile. } \\
\text { This clinical } \\
\text { presentation } \\
\text { forced a new } \\
\text { urgent medical } \\
\text { evaluation and } \\
\text { multiple } \\
\text { investigations at } \\
\text { the hospital, in } \\
\text { order to exclude } \\
\text { meningitis. }\end{array}$ & $\begin{array}{l}\text { Facing a negative } \\
\text { work-up for } \\
\text { meningitis, a } \\
\text { further assessment } \\
\text { by a dentist was } \\
\text { able to diagnose a } \\
\text { periodontal } \\
\text { abscess with local } \\
\text { neurological } \\
\text { symptoms. }\end{array}$ \\
\hline
\end{tabular}

\begin{tabular}{|c|c|c|c|c|}
\hline & What? & Main problem & Specific problem & $\begin{array}{l}\text { Improvement } \\
\text { proposals }\end{array}$ \\
\hline $\begin{array}{l}\text { What happened? } \\
\text { (main event) }\end{array}$ & Error & Diagnostic error. & $\begin{array}{l}\text { Missing diagnosis of } \\
\text { periodontal abscess; } \\
\text { Misinterpretation of } \\
\text { adverse events of } \\
\text { carbamazepine } \\
\text { (sleepiness). }\end{array}$ & \\
\hline \multirow[t]{3}{*}{$\begin{array}{l}\text { Why did it } \\
\text { happen? } \\
\text { (system failures) }\end{array}$} & Latent conditions & $\begin{array}{l}\text { Friendship with } \\
\text { patient; Lack of } \\
\text { teaching of oral } \\
\text { diseases to medical } \\
\text { doctors. }\end{array}$ & $\begin{array}{l}\text { Lack of differential } \\
\text { diagnosis; Lack of } \\
\text { consideration about } \\
\text { acute oral diseases. }\end{array}$ & $\begin{array}{l}\text { Assure a medical } \\
\text { evaluation through } \\
\text { a strictly } \\
\text { professional } \\
\text { relationship. } \\
\text { Improve medical } \\
\text { education in oral } \\
\text { disorders. }\end{array}$ \\
\hline & $\begin{array}{l}\text { Error-producing } \\
\text { conditions }\end{array}$ & $\begin{array}{l}\text { Cognitive bias } \\
\text { (anchoring and } \\
\text { confirmation bias). }\end{array}$ & $\begin{array}{l}\text { Insufficient clinical } \\
\text { assessment of the } \\
\text { patient. }\end{array}$ & $\begin{array}{l}\text { Implement case- } \\
\text { based supervision in } \\
\text { the emergency } \\
\text { setting. }\end{array}$ \\
\hline & $\begin{array}{l}\text { Faults in the } \\
\text { defenses }\end{array}$ & $\begin{array}{l}\text { Lack of clinical } \\
\text { guidelines for } \\
\text { patients presenting } \\
\text { with acute facial } \\
\text { pain. }\end{array}$ & $\begin{array}{l}\text { Absence of } \\
\text { evaluation by a } \\
\text { dentist for a patient } \\
\text { presenting with a } \\
\text { dental cause of } \\
\text { facial pain. }\end{array}$ & $\begin{array}{l}\text { Consider evaluation } \\
\text { by a dentist in every } \\
\text { facial pain to } \\
\text { establish dental or } \\
\text { non-dental causes. }\end{array}$ \\
\hline
\end{tabular}


35.

Improving Quality and Numbers of Error Reporting

C. Y. DiDonato

Self, Dudley, MA

The purpose of this study is to investigate the value of reporting error and to find methods that could promote and improve current error reporting concerns.

Statement of problem: In 2000 the Institute of Medicine published a landmark document To Err is Human, which launched a patient safety education and quality improvement initiative dedicated to reducing preventable medical errors. Error reporting systems have been created to gather error data with the intention of providing the opportunity to use the information as a vital part of discovering effective methods for error reduction. A lack of error reports and reports that lack clarity may be hindering error reduction strategy. Can error analysis find methods to improve and increase error reporting?

Description of the intervention or program: Introducing an error analysis class or curriculum to nursing education could improve the thought process and perception of patient safety in a non-punitive, constructive environment where errors can be analyzed and discussed.

Findings to date: Developing methods to prevent errors requires a recognition and comprehension of how and why errors occur before an effective plan of action can be implemented. It was determined that clinical knowledge deficits, lack of real time support, and poor communication were the top contributors to error. Errors are under reported when nurses fear the negative consequences of error reporting. With the exception of patients, the most vulnerable populations for error are nursing students and new graduates that do not have the clinical experience to recognize warning signs or indications that lead to error.

Lessons learned: Implications for practice include developing a curriculum or continuing education course dedicated to error analysis. The goal would be to develop and implement an error curriculum to improve awareness of error, the value of accurate and descriptive error reporting, and possible solutions to improve error rates. 
36.

\section{Mind the GAP - a Model for Remediating Clinical Reasoning}

J. Appel ${ }^{1}$, R. Pinto-Powell ${ }^{2}, \underline{\text { S. Gupta }}{ }^{3}$

${ }^{1}$ Wayne State University, Southfield, MI

${ }^{2}$ Dartmouth Medical School, Hanover, NH

${ }^{3}$ Rush University, Chicago, IL

Statement of problem: In an effort to develop clinical reasoning skills in our learners, a fundamental understanding of the theory, semantics and biases that drive the process of reasoning must be effectively mastered by the teachers entrusted with the responsibility of training learners at all stages. The Association of American Medical Colleges (AAMC) Clinical Skills report as well as the Institution of Medicine position statement addressed the importance of enhancing skills in diagnosis, with little prescriptive information on how best to do this.

Description of the intervention or program: Strengthening skills in clinical reasoning taps into the necessity to produce post-graduate trainees who practice safe, efficient and high value care. Developing and refining faculty skills is a challenging phenomenon in this time and resource-limited environment. We have developed a methodology to enhance the skills of faculty in recognizing and remediating clinical reasoning difficulties. It takes advantage of knowledge in the area of cognitive psychology (pattern recognition, hypothetico-deductive reasoning, dual-process theory), terminology (illness scripts, problem representation, etc.) and biases (anchoring, availability, premature closure, etc.) as described by Audetat. In addition, we have created an easy-to-recall tool for identification and for selecting remediation strategies: GAP (Gathering data, Appraising the data appropriately, Proclivity for bias). These tools are taught in an interactive workshop utilizing learner cases where participants demonstrate their use for diagnosis and management of learners at various stages of development. Findings to date: This faculty development skills workshop has been presented at a variety of regional and national locations, including the 2016 AAMC meeting and the 2018 Academic Alliance of Internal Medicine meeting. Formal feedback has been favorable. At AAMC, participants marked on a 5 -point Likert scale $(1=$ strongly disagree, $5=$ strongly agree) the extent to which they agreed with the following statements: the session was valuable (4.71), the facilitators were effective (4.50), and the objectives of the session were met (4.28). Faculty in particular valued it's reproducibility as a train-the-trainer tool. Feedback from the March 2018 AAIM meeting is pending.

Lessons learned: There is a continuing need for faculty development in this arena, specifically in disseminating commonly used terminology and the cognitive process behind clinical reasoning. Our workshops have been attended by faculty from all levels of medical education, highlighting the need for consistent reinforcement of a unified approach to clinical reasoning teaching, assessment and remediation strategies. A simplified framework, such as the GAP, may be of value to the busy clinician. This framework remains to be validated. 
37.

\title{
Supporting Trainees Involved in Medical Error
}

\author{
C. A. Sieniewicz \\ NHS England, Winchester, NH, United Kingdom
}

Statement of problem: Medical errors are common and unfortunately inevitable. Whilst caring for the patient affected must be the first priority, evidence suggests that being involved in or responsible for mistakes can have a profound and lasting effect on healthcare professionals and that many feel unsupported in the aftermath of the event. Many such incidents are not routinely discussed amongst peers and colleagues due to fear and stigma, limiting opportunities for support, learning and systems change.

Description of the intervention or program: Junior doctors within Wessex Deanery were invited to participate in focus groups to discuss how medical errors have affected them emotionally and professionally, what support they received within the organisation and how they felt support could be improved. Groups were facilitated by junior rather than senior clinicians to help participants discuss issues more freely without fear or judgement or implications for their future training. The dialogue within the groups was not audio- or video-recorded to allay concerns about confidentiality and data protection; instead, anonymised phrases and arising themes were documented throughout the discussions and shown to participants for their validation and approval at the end. The qualitative data gathered within groups was then analysed using thematic analysis, findings were discussed between both facilitators to ensure agreement and participants had the opportunity to review the findings and provide their input as to whether they feel their comments or sentiments had been misconstrued.

Findings to date: The information gathered provided insight into Wessex trainees' experiences of medical error, the support they received within Wessex and how support could be improved. This information was used to develop training and resources within Wessex to improve support available, with a view to fostering greater resilience, wellbeing and indirectly, employee retention. Participants found the experience of the focus groups beneficial, through shared support and understanding with peers and colleagues who have had similar experiences. It is hoped that the project may help trainees to talk more freely about medical error in the future, possibly helping to create a more open and blame-free culture where mistakes can be shared and learned from.

Lessons learned: Junior doctor focus groups can provide a valuable source of information as to how we may better support trainees involved in medical errors. Participation in such groups can be a beneficial experience for junior doctors, through shared support and understanding and fostering a more open and blame-free culture. 
38.

\title{
The Human Diagnosis Project. An Open Intelligence Program to Enhance Patient Diagnosis and Care
}

\author{
D. Pincus ${ }^{1,2}$, M. Rabner ${ }^{3}$, T. Brandon ${ }^{4}$ \\ ${ }^{1}$ Bond University, Gold Coast, Australia \\ ${ }^{2}$ Leading Steps Paediatric Clinic, Benowa, Australia \\ ${ }^{3}$ The Human Diagnosis Project, Washington, DC \\ ${ }^{4}$ Human Diagnosis Project, San Fransisco, CA
}

Statement of problem: Care for a patient involves multiple often disparate steps. A comprehensive approach from triage through diagnosis, treatment, discharge, and ongoing care prevention is necessary. Diagnosis is a critical early step in assessing, treating and managing health problems and is one of the most important potential error sources with regular adverse patient outcomes.

Description of the intervention or program: The Human Diagnosis Project (Human Dx) is a worldwide effort to build an open intelligence system that maps the steps to help any patient. The project is an open access, free and personalised medical education program that enables health workers to learn through interactive case based simulations that mimic clinical practice and assess multiple dimensions of clinical performance. The project combines the collective intelligence of physicians with machine learning to enhance clinical decision making. In less than 15 minutes, medical professionals around the world can share real clinical cases that are immediately converted into brief case-based simulations. These cases are available to the Human Dx community who are then able to solve the cases in 2-3 minutes and receive personalized feedback on their clinical reasoning. Human Dx builds on the science of learning to improve clinical decision making with repeated cycles of practice, feedback and reinforcement. Human Dx is being used as an assessment and training tool at many top medical institutions such as Harvard and Johns Hopkins. It has received contributions from over 8500 health professionals from 80 countries, 500 institutions and 80 specialties.

Findings to date: Preliminary data from Johns Hopkins shows that average accuracy, efficiency and overall performance increase as Human Dx learners solve more cases. Data also suggests that experienced physicians solving cases on the Human Dx platform demonstrates superior efficiency and accuracy performance compared to trainees. Using a combination of web-based technology, data analytics, and crowd-sourced practical cases, the Human Dx Project may have the requisite characteristics to serve as an objective diagnostic reasoning performance assessment tool.

Lessons learned: Human Dx has launched a personalised free medical education platform for any health worker. It has established a validated universal measure of clinician performance and health system quality. Health workers who solve rapid case simulations receive real time feedback on their clinical reasoning metrics including accuracy, efficiency and breadth. The program reaches over $40 \%$ of US internal medicine residency programs. Human Dx therefore not only enables machine learning into diagnostic reasoning but enhances Doctor's learning and patient care. 


\section{Clinical Vignette}

\section{Poster Session 2 - Clinical Vignettes}

40.

\section{A Disease That Mimics PMR and Must be Ruled out}

\section{Kurihara ${ }^{1}$, S. Kinjyo ${ }^{2}$, Y. Tokuda}

${ }^{1}$ Urasoe General Hospital, Okinawa, Japan

${ }^{2}$ Urasoe General Hosptal, Okinawa, Japan

${ }^{3}$ Okinawa Muribushi Project for Teaching Hospitals, Urasoe, Okinawa, Japan

Learning objectives: Polymyalgia rheumatica (PMR) is often recognized in elderly patients, but its diagnosis should be made by exclusion of other possible and important diseases.

Case information: A 78-year-old man presented with a 2-week history of severe bilateral shoulder pain. He visited an orthopedic clinic where MRI scan over the shoulder joints was taken, showing findings consistent with osteoarthritis of the shoulders. Thereafter, he gradually developed pain of bilateral upper limbs, along with weight loss and depression. The past medical history indicated that a diagnosis of pancreatic cancer was made several months ago, and the patient still underwent a series of chemotherapy. On examination, he was ill-appearing and afebrile. There was no joint swelling but tenderness over the bilateral upper limbs. Based on the Bird's criteria for PMR, a diagnosis of paraneoplastic PMR was made at first. Prednisolone was administered, but his symptoms did not improve. After admission, muscle weakness and numbness in bilateral upper and lower limbs developed. Cervical spine MRI scan was performed, showing multiple spine metastases of pancreatic cancer with spine cord compression in C4-6. He received urgent radiation therapy.

Discussion: Availability and confirmation biases might have been involved in this case, since PMR is common, the patient seemed to have its typical symptoms and no spine imaging tests were ordered initially. However, the diagnosis of PMR is challenging and careful differential diagnoses should also include infective endocarditis, seronegative RA, crowned-dens syndrome, hypothyroidism, or depression. In addition, there is no specific biomarker nor histopathological findings for PMR. Incessant critical review for the diagnosis should be sought when the improvement of symptoms in patients with PMR-like illnesses is poor. In particular metastatic cancer to the spine should be included into the do-not-miss diagnosis list in patients with history of active cancer. 
41.

\section{Great Mimicker: Whipple's Disease Presented As Rheumatoid Arthritis and Tuberculosis Lymphadenitis.}

\section{T. Nakajima ${ }^{1}$, Y. Oba ${ }^{2}$}

${ }^{1}$ Akashi Medical Center, Akashi, Japan

${ }^{2}$ Osaka General Medical center,, Osaka, Japan

Learning objectives: Gastrointestinal symptoms of Whipple's disease occur after joint pain. Anti-tuberculosis drugs may lead to diagnostic delay of Whipple's disease.

Case information: A 58 years old man with history of hemodialysis and diabetes mellitus presented to the clinic due to fever and joint pain. Rheumatoid arthritis was suspected and bucillamine was started. However, his symptoms did not improve for 6weeks. He was hospitalized in the other hospital and piperacillin/tazobactam was administered for seven days. His fever improved, but it recurred soon. He was referred to our hospital. Inguinal lymph nodes were swollen and biopsy revealed granulomatous lymphadenitis without acid fasted bacilli. We suspected tuberculous lymphadenitis and started anti-tuberculosis drugs. However, his fever prolonged. Four months after starting anti-tuberculosis drugs, numbness below the knees and loose stool occurred. His fever and arthralgia were worsened. Computer tomography revealed systemic lymphadenopathy. Then, we suspected Whipple's disease and performed biopsy of axillary lymph node. We found periodic acid schiff staining positive macrophage on the specimen (Figure1). We stopped anti-tuberculosis drugs and administered ceftriaxone for 2 weeks, followed by oral doxycycline for one year. Then, almost all of the symptoms soon diminished.

Discussion: Symptoms of classic Whipple's disease are gastrointestinal symptoms, arthralgia and weight loss. Gastrointestinal symptoms usually occur after joint pain. It may lead to diagnostic delay. In this case, we started rifampicin before performing biopsy, then PCR of Tropheryma whipplei was negative because rifampicin would be active for Tropheryma whipplei. After administering ceftriaxone and doxycycline, his fever and joint pain cured without recurrence. This clinical course is compatible to that of Whipple's disease. Whipple disease should be included in differential diagnosis when rheumatic arthritis and tuberculosis are suspected.

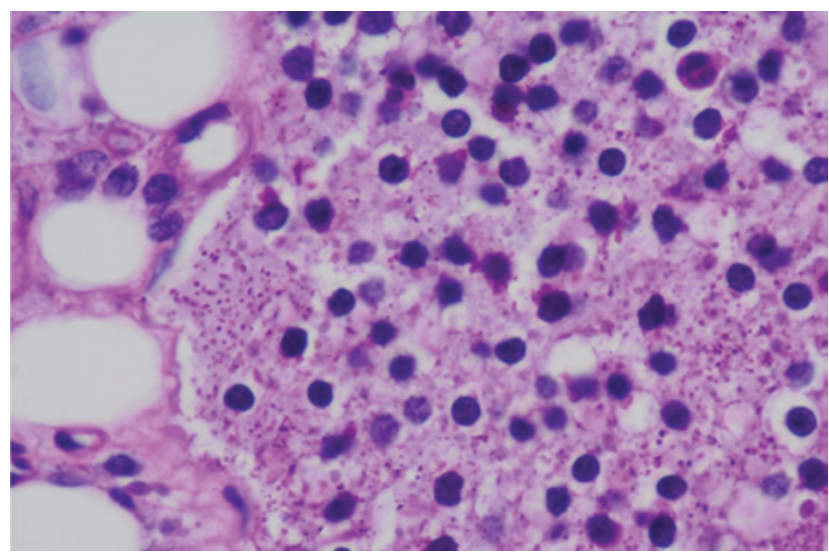


42.

\section{Hidden in Plain Sight}

\section{G. J. Jager ${ }^{1}$, M. Rutten ${ }^{2}$}

${ }^{1} J e r o e n$ Bosch Hospital, 's hertogenbosch, Netherlands

'Jeroen Bosch Hospital, 's-Hertogenbosch, Netherlands

Learning objectives: To understand why some obvious visible radiologic abnormalities are missed. To recognize which human factors contribute to these errors

Case information: Several radiologic cases with in hindsight obvious abnormalities, e.g. a surgical device not seen during initial examination due to inattentional blindness. Later during a grand round case discussion the device was not observed by 20 attendants. (Social context) Discussion: 'You see, but you do not observe. The distinction is clear.' Sherlock Holmes (A Scandal in Bohemia) It is estimated that one billion radiologic examinations are performed worldwide annually, with an estimated day-to-day error rate of 3-5\%. Most are subtle but a number are obvious. In this educational poster we present some missed radiologic findings, which are clearly seen in hindsight. The various physiological, cognitive, psychological and sociological mechanisms that contribute to these errors are discussed. These are perception, attention, recognition, memory, knowledge, (subconscious)thinking and reasoning including cognitive and affective biases, (selective) information and lack of time (bounded rationality) and social context. It is important for both radiologists and referring physicians to be aware and forearmed for these errors in order to prevent them.

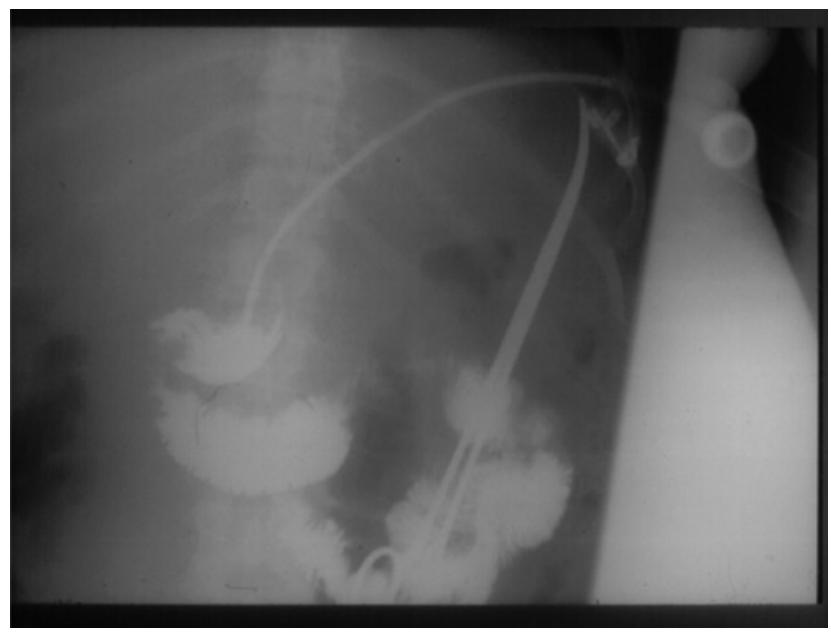


43.

Intravascular Lymphomatosis. Is It Possible to Diagnose Earlier? a Case Report

\author{
M. Song, M. Saijo, S. Constantine, U. Nakagawa
}

Sapporo Tokushukai Hospital, Sapporo, Japan

Learning objectives: Intravascular lymphomatosis (IVL) is a rare intravascular subtype of extra-nodal large cell lymphoma; with poor response to chemo/radiotherapy, and an extremely aggressive clinical course. The recognition of IVL in cases of fever of unknown origin (FUO) may help facilitate prompt treatment and improved outcomes.

Case information: An 80-year-old man with history of hypertension and polymyalgia rheumatica (PMR) presented to the emergency room (ER) with fever and chills. Five weeks prior, he had bilateral upper back pain associated with an elevated C-reactive protein; he was diagnosed with PMR and given low dose prednisone. On arrival to the ER, his physical exam was only remarkable for fever. His urinalysis showed pyuria, he was diagnosed with an urinary tract infection (UTI) and began receiving antibiotics. His fever persisted and it would break only with steroids. The urine culture grew Acinetobacter baumannii. Unfortunately, he developed diarrhea with positive Clostridium difficile toxin test. Pseudomembranous enteritis was treated with oral vancomycin but after 10 days, he became unconscious with a gradual hyperbilirrubinemia. We consulted a hepatologist, and the patient underwent liver biopsy. The patient died shortly after. The biopsy revealed intravascular lymphomatosis.

Discussion: Although IVL is a clonal proliferation of lymphocytes, it is uncommon to find enlarged lymph nodes, hepatosplenomegaly, or circulating cells in the peripheral blood. Fever is the most prominent sign in IVL in approximately $45 \%$ of cases. As in this case, an exhaustive search for an infectious etiology often contributes to the delay in diagnosis, which is frequently not established until autopsy. Including this disease in our differential diagnosis when other more common conditions have been excluded may lead to earlier diagnosis and may potentially decrease its lethality. However, in retrospect, we still wonder how we could have suspected IVL earlier in this man. 
44.

\section{Misdiagnosis of a Liver Abscess}

\section{K. Uehara ${ }^{1}$, Y. Harada ${ }^{2}$}

${ }^{1}$ Nagano Chuo Hospital, Nagano, Japan

${ }^{2}$ Dokkyo Medical University Hospital, Shimotsuga, Japan

\section{Learning objectives:}

1. To reconsider the diagnosis

2. To recognize abscesses as an etiology of fever

3. To distinguish discrepancy between cultures

Case information: An 83-year-old man visited the emergency department with a several-hour history of high-grade fever. His past medical history was remarkable for cerebral infarction, prostate cancer, diabetes mellitus, and atrial ventricular block. On physical examination, he was diaphoretic, but otherwise unremarkable. Laboratory studies revealed mild leukocytosis with neutrophilia and elevated transaminase levels. Urinalysis revealed pyuria and bacteriuria. Chest and abdominal non-contrast-enhanced computed tomography (CT) findings were normal, except for urinary retention. He was diagnosed with acute nephritis and intravenously administered with ceftriaxone after admission. On day 4, urine and blood cultures obtained at presentation tested positive for Pseudomonas aeruginosa and Klebsiella pneumoniae, respectively; thus, antibiotics were changed to ceftazidime. His condition and laboratory data improved promptly, and discharge was planned. The patient's physician was a resident and was unconcerned about the culture discrepancy. Therefore, he never suspected other fever etiologies. On day 12, however, a senior doctor indicated concern about the pyelonephritis diagnosis and advised the resident to perform further workup when he presented the case at a regular case conference. Abdominal contrast-enhanced CT revealed a 4-cm liver abscess, which could be suspected in the former abdominal CT. The therapeutic plan was changed for abscess treatment.

Discussion: Herein, misdiagnosis occurred. This is typically caused by anchoring and confirmation biases. The resident believed the initial diagnosis was correct and ignored inconsistent data of different microorganisms growing in blood and urine cultures, which are not usually observed in pyelonephritis patients; however, consultation with a senior doctor helped the resident eliminate bias. To reduce anchoring or confirmation bias-related diagnostic errors by inexperienced physicians, routine consultation with senior doctors can be beneficial, even when diagnoses are presumed correct. 
45.

\section{Misdiagnosis of Pericarditis with Fatal Consequences}

M. O. Van Aken ${ }^{1}$, G. J. Jager², L. Zwaan ${ }^{3}$

${ }^{1}$ Haga Hospital, The Hague, Netherlands

${ }^{2} J$ Jeroen Bosch Hospital, 's hertogenbosch, Netherlands

${ }^{3}$ Erasmus MC, Rotterdam, Netherlands

\section{Learning objectives:}

- Understand what we can learn from a case analysis of the medical disciplinary board

- Describe the variety of causes leading to a diagnostic error

Case information: The unexpected death of a 21-year old man in the Netherlands was widely covered in the media. We analyzed the factors that attributed to his death, based on the publicly available report of the medical disciplinary board. A young patient, until 2 days before admission in excellent physical shape, presented with acute onset complaints of dyspnea, painful shoulders and arms and general discomfort. Based on a chest X-ray, a pneumothorax was diagnosed and the patient was admitted with analgesics and i.v. saline. That night, the patient was found dead. Postmortem examination revealed a purulent peri- and epicarditis.

Discussion: What happened? This dramatic case highlights a variety of causes:

- Premature closure: The focus of the diagnostic process was on pneumothorax only. No differential diagnosis was considered.

- The triage note, stated that the patient "carried heavy load", which led to the working diagnosis of pneumothorax.

- The clinical presentation and lab results did not fit in a clinical scenario of pneumothorax, however, no further tests were performed. - When clinical deterioration occurred, there was no reassessment of the diagnosis.

- Misinterpretation: of (1) clinical situation/ severity, (2) a falsely observed pneumothorax on the first chest X-ray), (3) EKG (incomplete RBTB in young man is an abnormal finding that merits further investigation).

- Communication and handover: (1) the results of a second chest X-ray were not documented in the ER discharge letter. (2) The team on the ward stuck to the initial (wrong) diagnosis partly because of an incorrect hand-over.

- Organization: (1) The supervising pulmonologist was running an outpatient clinic on another hospital-site, and therefore unable to examine the patient himself. (2) The system for transferring a patient to another hospital-site was inadequate. 
46.

\section{Over the Overt Diagnosis}

$\underline{\text { S. Katsukura, Y. Harada, T. Shimizu }}$

Dokkyo Medical University Hospital, Shimotsuga, Japan

Learning objectives: To recognize the risk of overconfidence in the prior study results and expert's assessments.

Case information: A previously healthy 50-year-old woman presented to a neurology clinic with progressive numbness of the legs. While thoracic spinal cord lesion was suspected based on the neurologic examination, spinal cord magnetic resonance imaging (MRI) was normal; she was followed up with only observation. During following two months, however, her numbness exacerbated with difficulty in walking; she was referred to our diagnostic department for further investigation. Neurologic examination revealed decreased thermal and pain sensation bilaterally below the level of Th10 and vibratory sensation in the lower limbs. Laboratory studies revealed low vitamin B12 levels. At this point, any imaging tests were not performed based on the normal result of prior MRI with the prior neurologist's assessment. She was diagnosed with neuropathy caused by vitamin B12 deficiency, and treated with oral mecobalamin. Within next two months, however, her numbness at the bilateral level below Th4 progressed, and she became easier to fall. Spinal cord computed tomography and MRI revealed spinal cord compression by the ossification of the posterior longitudinal ligament and the yellow ligament at the Th1/Th2 level. She was diagnosed as thoracic myelopathy, and was admitted for an emergent operation.

Discussion: This case underscores the example of diagnostic error du to early closure and anchoring from overconfidence bias. Excessive dependence on "normal" imaging and prior expert's assessment swayed latter physicians from the diagnosis of spinal cord disease. In addition, other possible diagnosis, vitamin B12 deficiency, led to early closure in the diagnostic process. Clinician should recognize that even experts can miss the diagnosis of their realm especially at the early stage of the disease. Hence, it is important to revisit to repeat diagnostic studies even after "definitive" diagnosis is made, particularly when the patient's condition is deteriorating. 
47.

\section{Puzzling ECG, Unusual Cause}

\section{$\underline{\text { S. Swarup }}{ }^{1}$, M. L. Ebuna ${ }^{2}$}

${ }^{1}$ Khoo Teck Puat Hospital, SINGAPORE, Singapore

${ }^{2}$ Khoo Teck Puat Hospital, Singapore, Singapore

Learning objectives: One must consider common diagnosis before unusual ones, which goes along with the classical teaching of 'common things are common'

Case information: 25 year old policeman presented to the Emergency Department (ED) with an acute onset of breathlessness, giddiness, diaphoresis and palpitation. He was otherwise a fit male with an incidental diagnosis of mitral valve prolapse diagnosed on ECHO. His stress test done a year ago was normal.He was stable with no clinical findings. His initial ECG (electrocardiogram) showed broad complex regular tachycardia with AV dissociation. This was thought to be ventricular tachycardia. A consultation was sought with the cardiologist on-call. At this point, the patient was assumed to have fascicular ventricular tachycardia, a relatively benign condition. It was assumed that it would be unusual for a healthy male with no risk factors to have VT. The ECG showed a right axis pattern with relatively less broad QRS complex suggestive of FVT. He was given verapamil, the medication of choice for FVT. The patient soon had a syncopal episode, requiring synchronized cardioversion. His cardiac rhythm reverted to normal sinus rhythm and his condition stabilised. He was admitted to the Cardiology Intensive Care Unit. While in the unit he had a few more runs of VT. A diagnosis of cardiomyopathy with Cardiac Sarcoidosis was made after Cardiac MRI and tissue biopsy of his heart. The patient is currently on a left ventricular assisted device and is waiting for a heart transplant.

Discussion: Ventricular tachycardia is the commonest cause of a broad complex tachycardia and the most dangerous. Differential diagnosis are supraventricular tachycardia with aberrancy and fascicular ventricular tachycardia. Both are relatively benign with different management. When there is uncertainty, we should assume that common things are common and assume the worst.

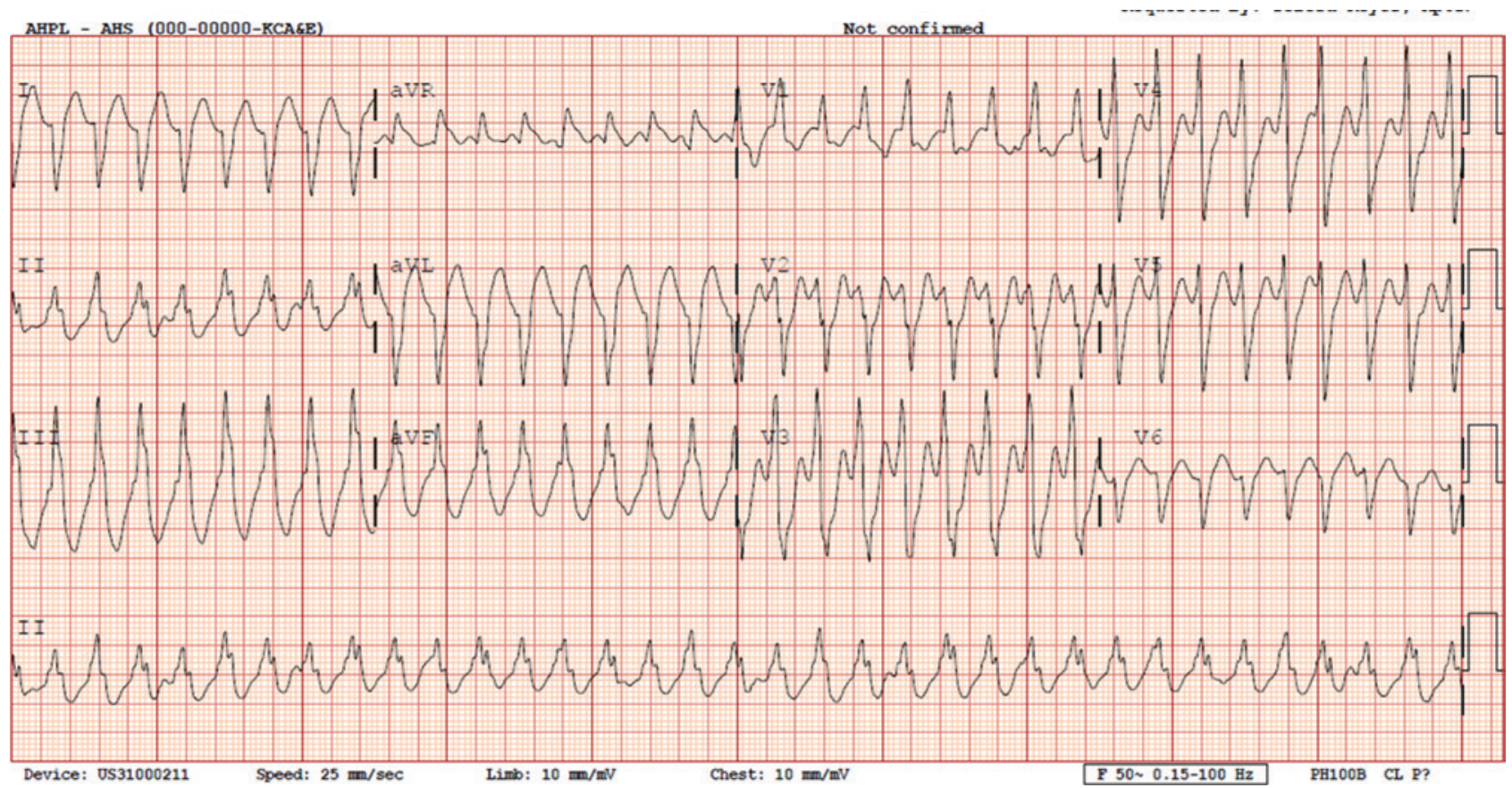


48.

\section{The Initial Findings of Synovial Fluid Analysis Delayed the Diagnosis of Septic Arthritis}

H. Baek, M. Saijo, S. Constantine, U. Nakagawa

Sapporo Tokushukai Hospital, Sapporo, Japan

Learning objectives: Septic arthritis(SA) should not completely be excluded despite moderate increase of leukocyte count, below 50,000 cells/mm3 in synovial fluid(SF) analysis. SF culture potentially prevent faulty synthesis.

Case information: 56-year-old woman with a history of hypertension and cerebellar infarction presented to the ED with knee pain and fever. On arrival, she was febrile with swelling, warmth and erythema of the right knee. SF was purulent, with leukocyte count of 15,200 cells/mm3 (mostly neutrophils). Even though no calcium pyrophosphate crystals were observed, pseudogout was still suspected due to the mild elevation of SF leukocyte count and the elevated level of C-reactive protein (CRP.) She rested in bed with oral loxoprofen (NSAID). Despite SF drainage and anti-inflammatories, her symptoms did not improve. On the hospital day 5, we repeated the arthrocentesis and found that the leukocyte count of the SF was now 198,750 cells/mm3, and the SF culture showed Streptococcus equisimilis. She began receiving antibiotics immediately, and she underwent arthroscopic knee lavage on hospital day 8. After 3 weeks of antibiotics therapy, she was discharged without further complications and in good condition.

Discussion: SA is a key consideration in adults presenting with acute monoarticular arthritis. Failure to initiate appropriate antibiotic therapy within the first 24 to 48 hours of onset can cause subchondral bone loss and permanent joint dysfunction. In SF analysis, a WBC count of more than 50,000 per mm3 and a polymorphonuclear cell count greater than 90 percent have been directly correlated with SA. Therefore, lower synovial fluid WBC counts have been reported in persons with disseminated gonococcal disease, peripheral leukopenia, or joint replacement. However, in this case, the patient did not have these problems, The comparatively low level of WBC lead to the delay of diagnosis. SA may have to be taken into consideration regardless of the initial low level of WBC count. 
49.

\section{“Look at Situation from All Angles, and You Will Become More Open”}

\section{S. Swarup ${ }^{1}$, E. Seow ${ }^{2}$}

${ }^{1}$ Khoo Teck Puat Hospital, SINGAPORE, Singapore

${ }^{2}$ Khoo Teck Puat Hospital, Singapore, Singapore, Singapore

Learning objectives: When assessing an elderly patient - avoid jumping to an early conclusion. It is not uncommon for the elderly patient to have an atypical presentation.

Case information: 70 year old Chinese lady comes to Emergency department with symptoms of giddiness, vomiting and diaphoresis. She was generally looking unwell morbid obese lady. On arrival she was afebrile had heart rate of 35 beats per minute, blood pressure of $65 / 55 \mathrm{~mm}$ $\mathrm{Hg}$ and good oxygenation. She was alert with no neurological deficit, normal heart and lung findings and soft abdomen. She had past medical history of hypertension, dyslipidaemia and ischemic heart disease. Initial ECG was suggestive of junctional bradycardia. She was managed for sick sinus syndrome, was treated with atropine and dopamine. Symptoms improved with improvement in vital parameters. Patient was planned admission under cardiology high dependency unit for cardiac pacing and monitoring. While awaiting for transfer, bed side ultrasound was performed to rule out other cause of hypovolemic shock, which was inconclusive. On further examination and while doing ultrasound abdomen, she complained of Left flank pain and tenderness. To evaluate further for left flank pain, CT scan was performed which showed ruptured aortic aneurysm retroperitoneal haematoma. She underwent urgent open graft repair of infra-renal aorta and post op was extubated the next day. She was discharged from hospital in stable and fit status.

Discussion: Abdominal aortic aneurysm (AAA) is a common and life threatening condition that affects $5-9 \%$ of the population over the age of 65 years. Rupture, the most common complication of AAA, is one of the most fatal surgical emergencies; it has an overall mortality rate of approximately $90 \%$. Misdiagnosis of ruptured AAA occurs in $30 \%$ of patients. This can only be prevented by considering the diagnosis and actively excluding it. Certain red-herrings can result in arriving at a premature diagnosis.

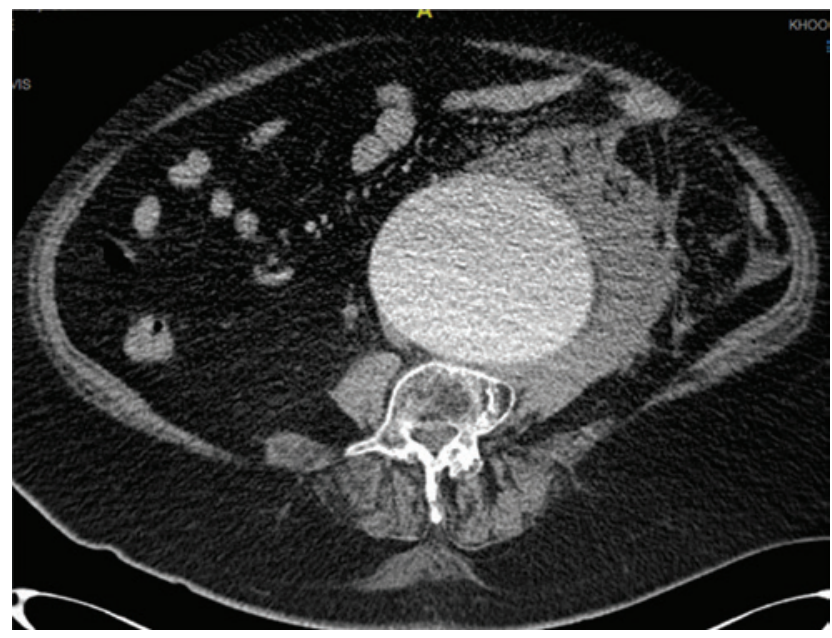

